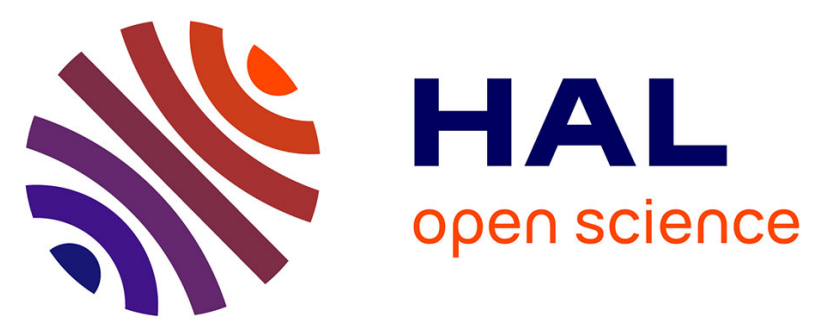

\title{
Mutations urbaines et logiques de localisation des emplois : le cas de la Communauté de l'Agglomération Dijonnaise (1990-1999)
}

Catherine Baumont, Françoise Bourdon, Rachel Guillain

\section{To cite this version:}

Catherine Baumont, Françoise Bourdon, Rachel Guillain. Mutations urbaines et logiques de localisation des emplois: le cas de la Communauté de l'Agglomération Dijonnaise (1990-1999). [Rapport de recherche] Laboratoire d'analyse et de techniques économiques(LATEC). 2003, 26 p., figures, tableaux, bibliographie. hal-01526533

\section{HAL Id: hal-01526533 \\ https://hal.science/hal-01526533}

Submitted on 23 May 2017

HAL is a multi-disciplinary open access archive for the deposit and dissemination of scientific research documents, whether they are published or not. The documents may come from teaching and research institutions in France or abroad, or from public or private research centers.
L'archive ouverte pluridisciplinaire HAL, est destinée au dépôt et à la diffusion de documents scientifiques de niveau recherche, publiés ou non, émanant des établissements d'enseignement et de recherche français ou étrangers, des laboratoires publics ou privés. 


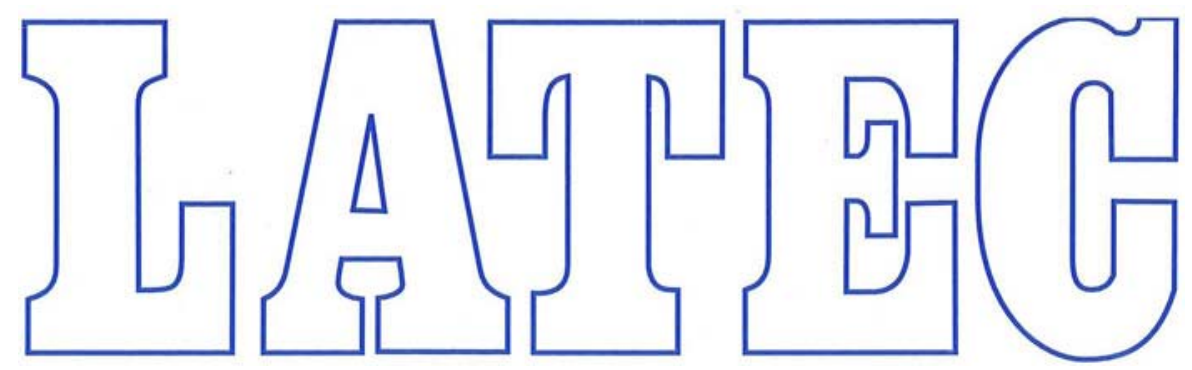

\section{LABORATOIRE D'ANALYSE ET DE TECHNIQUES ÉCONOMIQUES}

UMR 5118 CNRS

DOCUMENT DE TRAVAIL

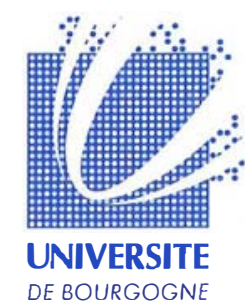

Pôle d'Économie et de Gestion

2, bd Gabriel - BP 26611 - F-21066 Dijon cedex - Tél. 0380395430 - Fax 0380395443

Courrier électronique : secretariat.latec@u-bourgogne.fr 


\begin{abstract}
$n^{\circ}$ 2003-13
Mutations urbaines et logiques de localisation des emplois : le cas de la Communauté de l'Agglomération Dijonnaise (1990-1999)

Catherine BAUMONT, Françoise BOURDON, Rachel GUILLAIN
\end{abstract}

septembre 2003 


\title{
MUTATIONS URBAINES ET LOGIQUES DE LOCALISATION DES EMPLOIS : LE CAS DE LA COMMUNAUTÉ DE L'AGgLOMÉRATION DIJONNAISE $(1990 ; 1999)$
}

\author{
Catherine Baumont, Françoise Bourdon et Rachel Guillain
}

e-mail : catherine.baumont@u-bourgogne.fr

francoise.bourdon@u-bourgogne.fr

rachel.guillain@u-bourgogne.fr

\begin{abstract}
Résumé :
L'objectif de cette recherche est d'étudier les mutations existant au sein de la configuration urbaine de la Communauté de l'Agglomération Dijonnaise, à travers notamment les nouvelles logiques de localisation des services supérieurs. Nous mettons alors en évidence la constitution de différents pôles d'emplois au sein de la COMADI, dont la composition économique diffère selon qu'il s'agit du pôle central ou des pôles périphériques, mais évolue aussi au cours de la période d'étude (19901999). Nous montrons que les tendances de localisation des activités de services supérieurs changent et se diversifient: si le centre historique reste spécialisé dans les activités financières, d'assurances et immobilières, les activités de services supérieurs aux entreprises tendent à se localiser davantage en périphérie. Une distinction est observée entre les deux pôles périphériques de la COMADI : au Sud, la constitution d'un pôle dédié aux activités industrielles et au Nord, la formation d'un pôle multifonctionnel.
\end{abstract}

\begin{abstract}
:
The aim of this paper is to analyze the spatial changes in the Dijon urban area (COMADI) by studying in particular the new tendencies of localization of the high order producer services. Our results display a multicentric structure of the COMADI which exhibits several employment poles. Not only the economic composition of these poles differs between the central pole and the peripheral ones but this composition changes over the study period (1990;1999). New localization logics appear for the high order services: if the historical center remains specialized in financial activities, insurance and real estate (FIRE), the other producer services are more likely to be localized in the periphery. A distinction has to be made between the two peripheral poles: the South pole is oriented toward industrial activities and the North one is more a multifunctional pole.
\end{abstract}

\footnotetext{
Mots clés : Centre-périphérie, multi-polarisation, services supérieurs, spécialisation, suburbanisation.
}

Classification JEL : L80, R12, R30

Cette étude s'intègre dans le programme de recherche du LEG « Analyse économique des nouvelles formes de suburbanisation dans les espaces urbains » soutenu par le contrat d'étude 20002002 de la Région Bourgogne "La recomposition urbaine dans la société de services et d'informations" (sous la direction de Catherine Baumont et Jean-Marie Huriot), le programme Aide à Projet Nouveaux 2000 du CNRS "Analyse économique des nouvelles formes de suburbanisation dans les villes françaises" (sous la direction de Catherine Baumont) et le programme DARES-PUCA "Services, emplois, territoires" du Ministère de l'Equipement, des transports et du logement "Services aux entreprises et nouvelles centralités urbaines" (sous la direction de Jean-Marie Huriot).

Nous remercions la Direction Régionale de l'INSEE Bourgogne et l'Agence Intercommunale d'Urbanisme Dijonnaise pour les différentes informations qu'ils nous ont fournies pour réaliser cette étude. 


\title{
POLARITÉS INTRA-URBAINES ET LOGIQUES DE LOCALISATION DES SERVICES : LE CAS DE LA COMMUNAUTÉ DE L'AGGLOMÉRATION DIJONNAISE $(1990 ; 1999)^{1}$
}

\author{
Catherine Baumont, Françoise Bourdon et Rachel Guillain
}

\section{INTRODUCTION}

Depuis une vingtaine d'années, les études empiriques urbaines mettent l'accent sur deux aspects essentiels du phénomène de recomposition des configurations urbaines : la multipolarisation de l'emploi urbain (BAUMONT et LE GALLO, 2000) et l'émergence de pôles tertiaires périphériques majeurs (BOITEUX-ORAIN et HURIOT, 2002). Les nouveaux modes de développement des territoires urbains sont illustrés par le modèle "Downtown-Edge Cities" (BINGHAM et KIMBLE, 1995; GARREAU, 1991) mis en évidence sur certaines grandes métropoles nord-américaines. Cependant, ce modèle répondant à des caractéristiques très spécifiques (surface de bureaux, de commerces de détail, flux journaliers de déplacements domicile-travail...), il semblerait que, pour bon nombre d'espaces urbains, une structure plus générale de type "pôle central (CBD) - pôles périphériques majeurs" s'applique. Au sein de ces nouveaux systèmes de centralités intra-urbaines, différents processus peuvent être observés tels que la spécialisation des pôles, le déclin du pôle central... qui conduisent à s'interroger sur les logiques de localisation centrale ou périphérique des activités économiques et sur les relations de concurrence ou de complémentarité unissant les différents pôles d'emplois.

De telles questions s'inscrivent dans un système économique marqué notamment par trois éléments : une dépendance toujours aussi forte aux moyens de transport, une dépendance devenue incontournable aux Nouvelles Technologies de l'Information et de la Communication et une croissance des activités de services supérieurs fortement créatrices de richesses. Dans ce contexte, les politiques d'aménagement et de développement économique des espaces urbains sont alors fortement interpellées. Où et comment développer les réseaux de transport et de communication et aménager les zones d'activités économiques ? Comment concilier l'attractivité du centre historique et celle des pôles périphériques et pour quelles activités ? ... Identifier les centres d'emplois et caractériser leur composition économique permettent de comprendre la formation et le fonctionnement des espaces urbains multipolarisés et peuvent éclairer ces choix de politiques urbaines.

Dans ce contexte, notre étude porte sur la recomposition économique de la Communauté de l'Agglomération Dijonnaise (COMADI) entre 1990 et 1999. Cet espace urbain français, cœur économique de la région Bourgogne, est la plus grosse agglomération française située à égale distance entre l'agglomération parisienne et l'agglomération lyonnaise. Il reste cependant de taille modeste comparé aux métropoles urbaines généralement étudiées. Il peut néanmoins être l'objet de mutations comparables car il peut engager, comme l'ensemble des agglomérations françaises, les mêmes types de politiques urbaines de développement et parce qu'il est soumis, comme l'ensemble des villes occidentales, aux mêmes types de mutations

\footnotetext{
${ }^{1}$ Certaines parties de cet article ont été présentées au $39^{\text {ème }}$ colloque de l'ASRDLF "Concentration et ségrégation, Dynamiques et inscriptions territoriales", Université Lyon II et ENTPE, Lyon, 1-3 septembre 2003, et à la $13^{\mathrm{e} m e}$ conférence internationale du RESER "Services et développement régional", FUCAM, Mons (Belgique), 9 et 10 octobre 2003.
} 
économiques. Dans cet esprit, nous nous interrogeons plus particulièrement sur les logiques de localisation des activités tertiaires et analysons quels sont, parmi les processus énoncés plus haut, ceux qui peuvent caractériser le territoire dijonnais. Après avoir identifié les polarités intra-urbaines au sein de la COMADI (I), nous étudions les mutations qui caractérisent la configuration urbaine : phénomènes de suburbanisation des activités, processus de spécialisation des pôles d'emplois, déclin potentiel du pôle central ... (II). Nous étudions ensuite, de manière plus approfondie, les logiques de localisation des activités de services supérieurs (III) et soulignons, en conclusion, les principales tendances d'évolution de la configuration urbaine dijonnaise.

\section{LES POLARITÉS INTRA-URBAINES AU SEIN DE LA COMADI}

Avec une surface de $172,4 \mathrm{~km}^{2}$, une population de 238309 habitants et près de 120000 emplois en 1999, la COMADI reste un territoire urbain de petite taille comparé à ceux généralement analysés dans les études urbaines sur la multicentricité (villes nord-américaines, grandes villes européennes, asiatiques ou des PVD). Néanmoins, la COMADI, comme la plupart de ces espaces urbains, est caractérisée par une organisation multipolarisée de ses activités économiques.

Cette organisation multipolarisée est liée à un ensemble de dispositifs d'aménagement des territoires urbains touchant à la fois l'organisation interne des villes et les modalités de fonctionnement de structures intercommunales. D'un côté, les communes françaises ont mis en place à partir des années 1960, deux types de politiques urbaines d'aménagement pour soutenir et développer l'attractivité économique de leur territoire en créant de nouveaux sites d'accueil ou en renforçant les sites existants. Il s'agit, d'une part, de la création, en périphérie des villes de nombreuses zones industrielles et commerciales auxquels ont plutôt succédé dans les années 1980 les parcs tertiaires et technologiques. Il s'agit, d'autre part, des politiques de rénovation et d'aménagement des quartiers centraux, souvent associées aux plans de sauvegarde des centres-villes, qui permettent de maintenir, voire de développer, l'attractivité du centre historique. D'un autre côté, différents dispositifs de regroupements de communes se sont succédés dans la seconde moitié du $20^{\text {ème }}$ siècle pour améliorer le fonctionnement des espaces urbains. Dans un tel contexte, la nature de la configuration intercommunale des emplois (multipolaire ou centre-périphérie) peut se superposer aux schémas centre-périphérie internes aux communes.

Notre étude de la COMADI s'intéresse à cette double dimension - intra et inter communales des logiques de localisation des activités économiques. Pour cela, il faut disposer de données recueillies à une échelle intra-urbaine. Après avoir présenté la base de données originale que nous avons utilisée (1.1.), nous précisons la méthode d'identification des centres que nous avons mise en œuvre (1.2.) pour révéler les polarités intra-urbaines de la COMADI (1.3.).

\subsection{La COMADI : caractéristiques et données utilisées}

La COMADI se compose actuellement de 16 communes contiguës : la ville-centre Dijon et 15 autres communes banlieues encerclant Dijon (cf. Carte $\mathrm{n}^{\circ} 1$ ): Ahuy, Chenôve, ChevignySaint-Sauveur, Daix, Fontaine-lès-Dijon, Longvic, Marsannay-la-Côte, Neuilly-lès-Dijon, Ouges, Perrigny-lès-Dijon, Plombières-lès-Dijon, Quetigny, Saint-Apollinaire, Sennecey-lèsDijon et Talant. Par rapport au zonage défini par l'INSEE (LE JEANNIC, 1998), elle représente, à une commune près (Ahuy), le pôle urbain dijonnais structurant l'aire urbaine dijonnaise. Entre 1990 et 1999, cette aire urbaine est passée de 163 à 214 communes et, alors que la COMADI couvre seulement $8 \%$ de l'aire urbaine en 1999 , elle concentre $73 \%$ de sa population et $86 \%$ de l'emploi. (cf. Tableau $\mathrm{n}^{\circ} 1$ ). 


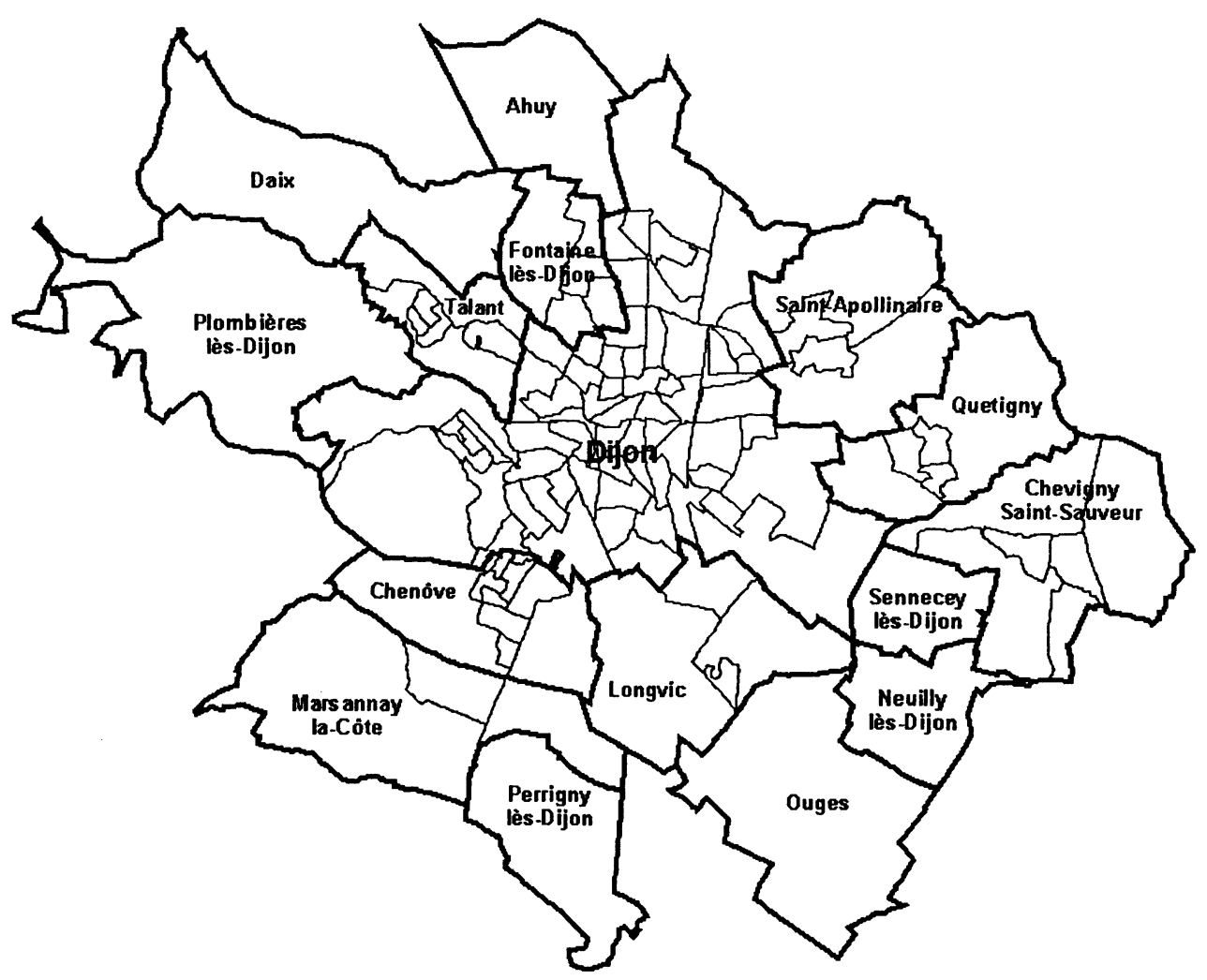

Tableau $n^{\circ} 1$ : La COMADI en quelques chiffres

\begin{tabular}{lcc}
\hline \multicolumn{1}{c}{ Caractéristiques } & COMADI & Aire Urbaine dijonnaise \\
\hline Surface totale & $172,4 \mathrm{~km}^{2}$ & $2271 \mathrm{~km}^{2}$ \\
Nb de communes (1999) & 16 & 214 \\
Population (PSDC) 1990* & 231734 & \\
Population (PSDC) 1999* & 238309 & 326887 \\
Population active 1990* & 108500 & \\
Population active 1999* & 111028 & 153401 \\
Emploi 1999* & 119838 & 138938 \\
Emploi salarié 1990** & 74493 & \\
Emploi salarié 1999** & 70770 & 82958 \\
\hline
\end{tabular}

Sources : calculs effectués par les auteurs d'après les données *RGP et**SIRENE de L'INSEE.

Les données utilisées concernent l'emploi et la population totale sans double compte au recensement de 1990 et 1999. Elles proviennent des fichiers du Recensement de la Population et des fichiers SIRENE fournis par la Direction Régionale Bourgogne de l'INSEE pour l'ensemble des communes de la Bourgogne. Ces données sont identifiées à la fois au niveau communal et à un niveau infra-communal (IRIS-2000 ${ }^{\circledR}$ ) pour les communes de plus de 5000 habitants (cf. Annexe $\mathrm{n}^{\circ} 1$ ). Parmi les 16 communes de la COMADI, 9 communes sont irisées : Chenôve (9 IRIS), Chevigny-Saint-Sauveur (5), Dijon (66), Fontaine-lès-Dijon (5), Longvic (4), Marsannay-la-Côte (3), Quetigny (5), Saint-Apollinaire (4) et Talant (6) (cf. Carte $\mathrm{n}^{\circ} 1$ ). Notre échantillon comprend au total 114 unités spatiales d'observations (cf. Annexe $n^{\circ} 2$ ) pour lesquelles nous disposons d'un ensemble de données démographiques (population), économiques (nombre d'établissements et effectifs salariés répertoriés selon les 
nomenclatures NAP600 pour 1990 et NAF700 pour 1999) et géographiques (surface et coordonnées des centroïdes) ${ }^{2}$.

Concernant l'exploitation des données d'emplois issues des fichiers SIRENE, trois éléments sont à souligner : d'abord, ces données correspondent à l'emploi salarié et sont très imparfaites notamment sur l'emploi public, ensuite, les procédures d'irisage des données nécessitent d'avoir une localisation suffisamment précise de l'établissement ce qui n'est pas toujours le cas et enfin, un changement de nomenclature des activités économiques est intervenu entre 1990 et 1999. Dans ces conditions, nous ne nous intéressons dans notre étude qu'aux effectifs salariés du secteur privé des établissements de 1 salarié et plus et nous avons ré-harmonisé les données de 1990 par rapport à la nomenclature NAF700 afin de construire les différents soussecteurs d'activités utilisés dans cette recherche. Notons qu'une structure plus complète de l'emploi peut être fournie par l'exploitation des données d'emplois au lieu de travail du RGP. A titre de comparaison, en 1999, l'emploi au lieu de travail recensé s'élève à 119 838, dont 42 629 emplois salariés du secteur public et 8074 emplois non salariés. Ne disposant pas de ces données ni à l'échelle IRIS ni selon une nomenclature d'activités nous permettant de distinguer les différentes activités de service pour les deux années, nous avons préféré exploiter les données SIRENE.

Deux traits principaux caractérisent l'organisation spatiale générale de l'emploi et de la population au sein de la COMADI, aussi bien en 1990 qu'en 1999 (BAUMONT et BoURDON, 2002). En premier lieu, la répartition spatiale de l'emploi est fortement concentrée puisque $25 \%$ des emplois se regroupent sur seulement $6 \%$ du territoire, que $50 \%$ des emplois occupent 15 à $16,4 \%$ du territoire et que seuls 29 à $34 \%$ du territoire suffisent à capter $75 \%$ des emplois ${ }^{3}$. En second lieu, on remarque une organisation spatiale radioconcentrique des 114 zones selon leurs niveaux ou leurs densités d'emploi et de population et ceci aussi bien en 1990 et en 1999. Les IRIS centraux sont plutôt très riches en emplois et en population et peuvent être qualifiés d'Integrated Districts au sens de FUJITA et OGAWA (1982). La couronne intermédiaire est à dominante résidentielle tandis que la couronne périphérique présente une structure asymétrique. A l'Est de l'agglomération, elle se compose surtout de zones périphériques plutôt riches en emplois et pauvres en population (zones industrielles, zones commerciales, Parcs tertiaires), tandis que les zones périphériques de l'Ouest sont pauvres en emplois et en population (Communes et Iris périphériques résidentiels). Néanmoins, au-delà de ce constat global, une étude plus approfondie de la localisation de l'emploi va nous permettre de situer et de caractériser les principaux pôles d'emplois de la COMADI.

\subsection{Méthodologie de l'identification des pôles d'emplois}

Dans les études empiriques sur la multipolarisation des espaces urbains, la méthode d'identification des pôles d'emplois de GIULIANO et SMALl (1991) est largement employée (BAUMONT et LE GALlO, 2000). Pour ces auteurs, un centre ou pôle d'emplois est une zone ou un ensemble de zones contiguës qui possèdent individuellement une densité d'emplois supérieure un certain seuil $\bar{D}$ et supérieure à la densité des zones qui l'entourent et qui possèdent globalement un nombre d'emploi total supérieur à un certain seuil $\bar{E}$. Cette définition reste largement empirique et nécessite de fixer des valeurs pour les seuils ainsi qu'un critère de contiguiité. On pressent par exemple que la valeur du seuil d'emploi dépendra

\footnotetext{
${ }^{2}$ Les fonds de carte à l'échelle IRIS ont été construits par Julie Le Gallo à partir de fonds de cartes à l'îlot fournis par la Direction Régionale Bourgogne de l'INSEE. Les données cartographiques ont été traitées à l'aide du logiciel Arc-ViewC3.2.

${ }^{3}$ Les valeurs des indices de Gini calculés sur les séries d'emplois et de surface sont respectivement de 0,77 pour 1990 et de 0,745 pour 1999.
} 
de l'espace urbain étudié (est-ce raisonnable d'utiliser pour la COMADI, le même seuil que pour Los Angeles : $\bar{E}=10000$ emplois ?) et des caractéristiques de l'urbanisation qui peuvent par exemple différer d'un continent à l'autre. Cependant, quel que soit cet espace, le nombre de pôles sera décroissant avec la valeur des seuils d'emplois et de densité. Cette méthode donne également un fort poids à la mesure de densité alors que l'utilisation d'un critère de densité brute ou de densité nette est susceptible d'induire des résultats différents suivant la finesse du découpage spatial disponible. D'autres auteurs préconisent alors l'utilisation du quotient de localisation (BOITEUX-OraIN et GUILLAIN, 2003 ; GASCHET, 2000 ; ForsTALL et GREEN, 1997) ou du ratio emploi-population (MC DONALD, 1987). Cette méthode a ainsi fait l'objet de plusieurs aménagements selon la taille de l'espace urbain étudié, selon ses caractéristiques et selon la nature du découpage spatial de manière à mieux adapter son pouvoir exploratoire à l'espace étudié.

Pour notre étude, deux éléments nous ont semblé particulièrement importants à considérer : la taille de la COMADI et la juxtaposition de deux échelles spatiales (IRIS et communes) au sein de l'échantillon. A notre connaissance, aucune étude d'identification des pôles d'emplois sur un espace urbain de taille modeste n'a été réalisée selon la méthodologie de Giuliano et Small. Dans ce cas, nous ne pouvions pas nous référer à un seuil d'emploi global adapté à des espaces de petite taille. Par ailleurs, le découpage spatial dont nous disposons crée une forte hétérogénéité spatiale (BAUMONT et al., 2003) sur le territoire étudié puisque nous disposons à la fois de deux types d'échelles : l'IRIS et la commune et que la construction même des IRIS n'est pas homogène selon qu'il s'agit d'IRIS d'emplois ou de population (cf. Annexe 1). En conséquence, les unités spatiales sont très petites au centre des communes irisées, elles sont plus grandes pour les IRIS d'activités économiques en périphérie de ces mêmes communes et enfin, elles sont encore relativement plus grandes pour les unités spatiales communales, souvent situées à la périphérie de la COMADI (cf. Carte $\mathrm{n}^{\circ} 1$ ). Dans ces conditions, appliquer un critère de densité à l'ensemble du territoire ne nous semblait pas adapté et pour cerner a priori cette forme d'hétérogénéité spatiale, nous avons alors distingué les unités spatiales selon leur taille $\bar{t}_{i}$. Nous avons également préféré utiliser un critère de seuil d'emploi individuel $\bar{e}_{i}$, comme le préconisent d'autres auteurs (BOITEUX-ORAIN et GUILLAIN, 2003 ; GASCHET, 2000) et avons appliqué le critère de densité $\bar{d}_{i}$ aux unités spatiales de petite taille tandis que l'on a utilisé pour les unités spatiales plus grandes le ratio emploi-population $\bar{r}_{i}$.

Une analyse de la distribution de la taille des unités spatiales montre que les IRIS de population ont une taille inférieure à 203 acres et ce seuil nous permettra donc de distinguer les unités spatiales de petite taille des autres. Le seuil de densité d'emplois est fixé à 8 emplois par acre (cette valeur oscille entre 15 et 6 pour les études empiriques sur les aires urbaines nord-américaines) et le seuil du ratio emploi-population est fixé à 1 . Le seuil individuel d'emplois est quant à lui fixé à 1000 . A titre de comparaison, GASCHET (2000) utilise un seuil de 2000 emplois pour l'agglomération bordelaise et BOITEUX-ORAIN et GUILLAIN (2003) retiennent un seuil de 5000 emplois pour l'Ile-de-France.

Finalement un pôle d'emplois sera constitué par une zone $i$ ou un ensemble de zones $i$ contiguës satisfaisant les critères suivants (Figure $\mathrm{n}^{\circ} 1$ ) : 
Figure $n^{\circ} 1$ : Les critères d'identification des pôles d'emploi

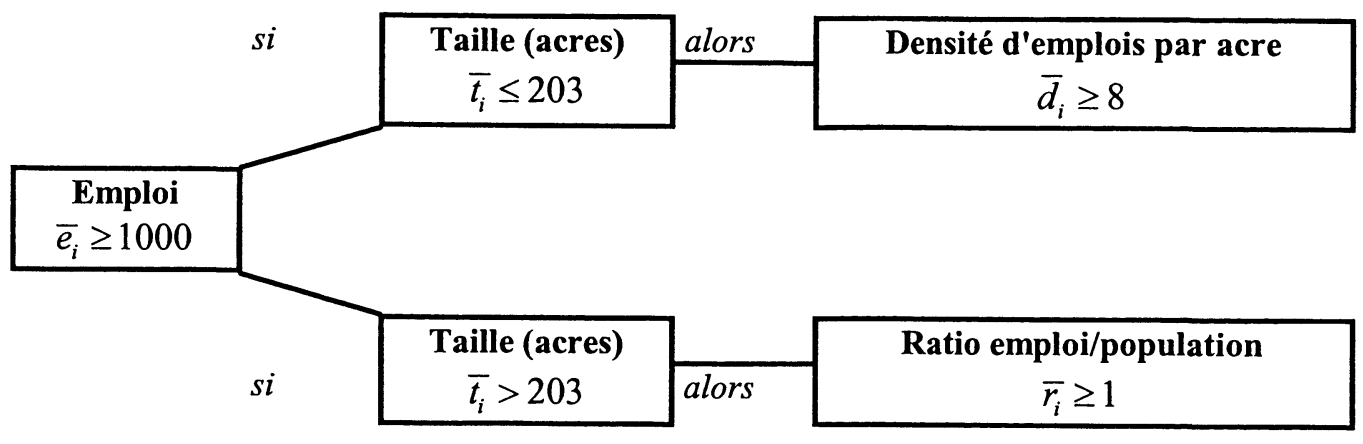

\subsection{La configuration urbaine de la COMADI}

L'identification des pôles d'emplois de la COMADI est réalisée en appliquant cette méthode aux données de 1999. Vingt zones satisfont les différents critères. Elles concentrent $65 \%$ de l'emploi de la COMADI et occupent $25 \%$ de sa surface totale. Dans cet ensemble, le regroupement des zones contiguës aboutit à la constitution de 5 pôles d'emplois dont la localisation (cf. Carte $\mathrm{n}^{\circ} 2$ ) et la composition sont données ci-après (cf. Tableau n ${ }^{\circ} 2$ ).

\section{Carte $n^{\circ} 2$ : Les pôles d'emploi dans la COMADI}

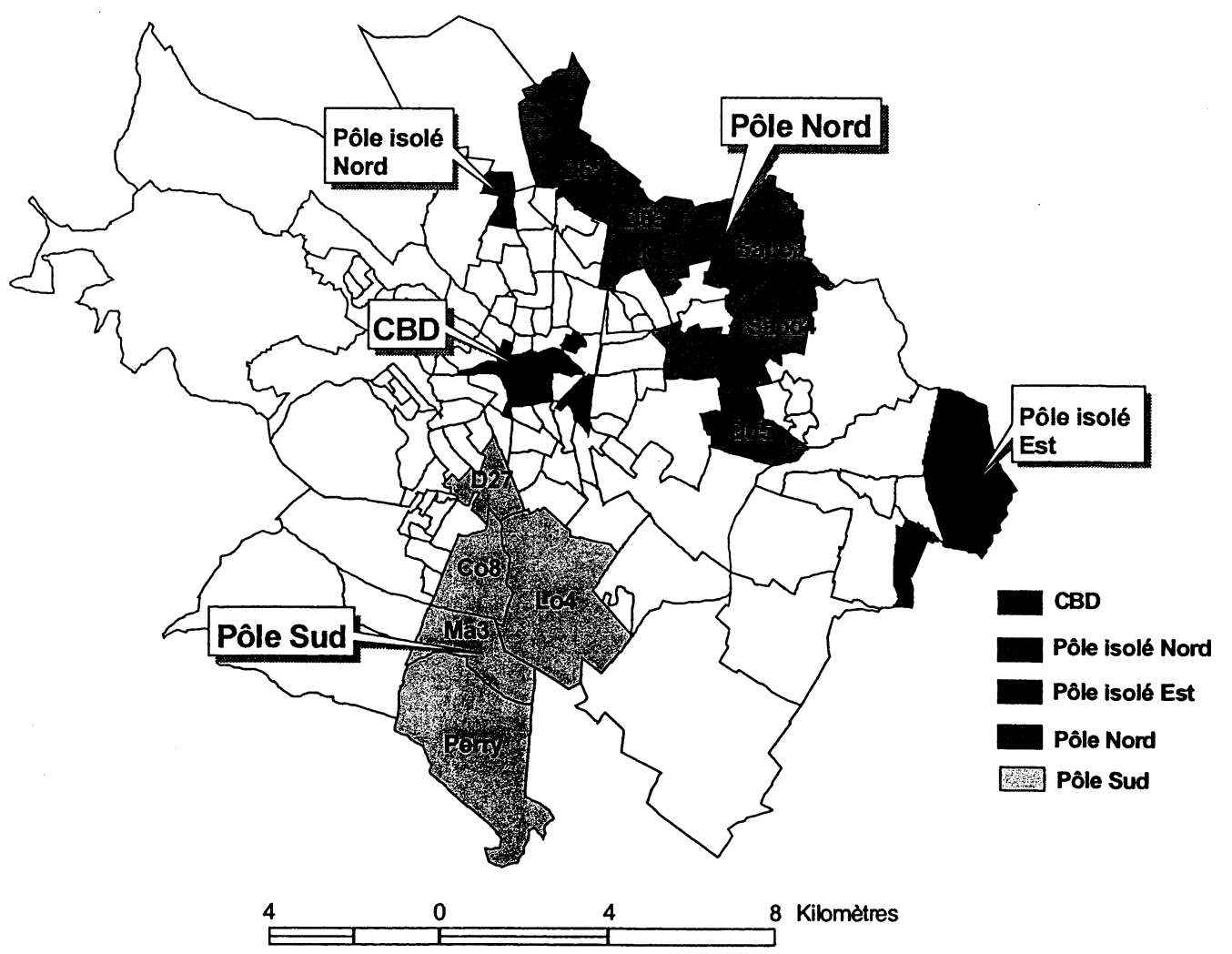

On distingue trois natures de pôles d'emplois. En dehors du CBD qui est le centre d'emplois historique, on trouve deux pôles périphériques qualifiés de multi-communaux car ils 
s'étendent sur le territoire de plusieurs communes. Enfin, les pôles isolés sont constitués d'une seule zone. Ils sont alors de taille beaucoup plus modeste et en croissance d'emplois sur la période. On peut remarquer que trois pôles d'emplois satisfont le critère de seuil d'emploi total ( $\bar{E}=10$ 000) utilisé par GIULIANO et SMALl (1991) dans leur étude de l'aire métropolitaine de Los Angeles. Les grands pôles sont de taille similaire en 1999 (entre 14000 et 15000 emplois), mais ont suivi des trajectoires de croissance différentes au cours de la période. Pour les deux années, le CBD est le pôle d'emplois le plus important, mais il perd des emplois entre 1990 et 1999 (-3 113 emplois), tandis que le pôle périphérique Nord voit sa taille augmenter (+2 824 emplois) et que le pôle périphérique Sud perd 1590 emplois.

Par ailleurs, on peut souligner deux traits de l'occupation spatiale de l'emploi sur ce territoire. Il s'agit tout d'abord du caractère asymétrique de cette occupation puisque aucun pôle d'emplois ne se localise à l'Ouest de l'agglomération. Ensuite, les pôles d'emplois se situent le long de deux grands axes de circulation : l'axe transversal Nord-Sud, d'une part, et la rocade périphérique $\mathrm{Est}^{4}$, d'autre part. La localisation des pôles isolés renforce cette image. Pour la COMADI comme pour l'essentiel des territoires urbains, la proximité des grands axes de transport, gage d'accessibilité, reste alors un facteur explicatif majeur de la structuration spatiale de l'emploi.

Tableau $n^{\circ} 2$ : Composition des pôles d'emplois de la COMADI

\begin{tabular}{|c|c|c|c|}
\hline \multirow[t]{2}{*}{ Nom et Caractéristiques } & \multirow[t]{2}{*}{ Composition } & \multicolumn{2}{|c|}{ Emploi total } \\
\hline & & 1990 & 1999 \\
\hline CBD & Monge (D1); Cordelier (D2); Grangier (D4) & 18270 & 15157 \\
\hline \multirow[t]{3}{*}{ Ville-Centre (Dijon) } & Jean-Jacques Rousseau (D5); Darcy (D6) & & \\
\hline & Clemenceau (D9)*; Voltaire (D14) & & \\
\hline & La Gare (D64) & & \\
\hline \multirow{5}{*}{$\begin{array}{l}\text { Pôle Sud } \\
\text { «Multi-communes » }\end{array}$} & ZAE de Chenôve (Co8) & 15302 & 13712 \\
\hline & Arsenal (D27 ; Dijon) & & \\
\hline & ZAE de Longvic (Lo4) & & \\
\hline & ZAE de Marsannay-la-Côte (Ma3) & & \\
\hline & Perrigny-lès-Dijon (Perry) & & \\
\hline \multirow{5}{*}{$\begin{array}{l}\text { Pôle Nord } \\
\text { «Multi-communes » }\end{array}$} & ZAE de la Toison d'Or (D62)* & 12008 & 14832 \\
\hline & ZAE Nord-Est de Dijon (D63) & & \\
\hline & ZAE de Quetigny (Qu5) & & \\
\hline & ZAE Nord-Est de Saint-Apollinaire (Sapo3) & & \\
\hline & ZAE Sud-Est de Saint-Apollinaire (Sapo4) & & \\
\hline $\begin{array}{l}\text { Fontaine } \\
\text { «Pôle isolé » Nord }\end{array}$ & ZAE de Fontaine-lès-Dijon (Fo5)* & 942 & 1088 \\
\hline Chevigny & ZAE de Chevigny-Saint-Sauveur (Ch5) & & \\
\hline «Pôle isolé» Est & & 1357 & 1421 \\
\hline
\end{tabular}

* ces zones ne vérifiaient pas le seuil de 1000 emplois en 1990.

$\mathrm{Si}$ on analyse les caractéristiques générales des 3 principaux pôles d'emplois, plusieurs éléments ressortent (cf. Tableau $\mathrm{n}^{\circ} 3$ ). Le $\mathrm{CBD}$ est un pôle de petite taille qui couvre seulement $1,13 \%$ du territoire de la COMADI. Il se caractérise par des densités élevées de l'emploi et de la population. Celles-ci sont de l'ordre de 9500 habitants par $\mathrm{km}^{2}$ et en augmentation sur la période : le CBD voit sa population croître de 8,6\% entre 1990 et 1999 ,

\footnotetext{
${ }^{4}$ Il n'existe pas pour l'instant de rocade périphérique Ouest : la LINO (Liaison Nord-Ouest) sera réalisée dans les prochaines années.
} 
alors que la croissance de la population de l'agglomération n'est que de $2,83 \%$ sur cette même période. Les densités sont, au contraire, en forte diminution pour l'emploi passant de 9350 emplois par $\mathrm{km}^{2}$ en 1990 à 7757 emplois par $\mathrm{km}^{2}$ en 1999. Ce chiffre traduit pour le CBD une perte d'emplois de $17 \%$ (soit 3113 emplois) alors que la COMADI ne perd que $4,8 \%$ de ses emplois salariés du secteur privé sur cette période ${ }^{5}$. Le pôle central concentre par ailleurs plus du quart des établissements de la COMADI et ces établissements sont de petite taille (environ 7 emplois par établissement).

Les deux pôles périphériques possèdent des caractéristiques opposées à celles du CBD : de tailles plus importantes (de l'ordre de $10 \%$ du territoire de l'agglomération dijonnaise chacun), leurs densités d'emplois sont de l'ordre de 800 emplois au $\mathrm{km}^{2}$ sur la période. Composés de Zones d'Activités Economiques, les densités de population y sont très faibles. Ces pôles périphériques regroupent de 6 à $9 \%$ des établissements de la COMADI qui sont plutôt des établissements de grande taille : de l'ordre de 23 emplois par établissement en 1990 à environ 18 emplois par établissement en 1999. Sur la période et dans le contexte global d'une diminution de l'emploi salarié privé sur la COMADI, on note que le pôle périphérique Nord gagne des emplois $(+23,5 \%)$, tandis que le pôle périphérique Sud perd des emplois $(-10,4 \%)$, alors qu'ils sont sensiblement de la même taille $\left(17 \mathrm{~km}^{2}\right)$.

Tableau $n^{\circ} 3$ : Caractéristiques des pôles d'emplois de la COMADI

\begin{tabular}{lrrrrrrrrr}
\hline & CBD & Pôle Nord & Pôle Sud & Fontaines & Chevigny & \multicolumn{1}{c}{ Total } \\
pôles & \multicolumn{2}{c}{$\begin{array}{l}\text { Hors } \\
\text { Pôles }\end{array}$} & COMADI \\
\hline SURF(\%) & 1,13 & 9,65 & 10,69 & 2,77 & 0,29 & 24,54 & 75,46 & 100 \\
EMP90 & 18270 & 12008 & 15302 & 1357 & 942 & 47879 & 26454 & 74333 \\
EMP99 & 15157 & 14832 & 13712 & 1421 & 1088 & 46210 & 24560 & 70770 \\
$\Delta$ EMP(\%) & $-17,04$ & 23,52 & $-10,39$ & 4,72 & 15,50 & $-3,49$ & $-7,16$ & $-4,79$ \\
$\Delta$ POP(\%) & 8,61 & 2,31 & 0,11 & 5,26 & $-10,84$ & 6,85 & 2,40 & 2,83 \\
DPOP90* & 9042 & 57 & 204 & 16 & 163 & 533 & 1608 & 1344 \\
DPOP99* & 9821 & 58 & 204 & 17 & 146 & 569 & 1647 & 1382 \\
DEMP90* & 9350 & 722 & 830 & 285 & 1854 & 1132 & 203 & 431 \\
DEMP99* & 7757 & 892 & 744 & 298 & 2142 & 1092 & 189 & 411 \\
ETAB90 & 2453 & 505 & 650 & 39 & 54 & 3701 & 4980 & 8681 \\
ETAB99 & 2214 & 830 & 770 & 56 & 59 & 3929 & 4875 & 8804 \\
DETAB(\%) & $-9,74$ & 64,36 & 18,46 & 43,59 & 9,26 & 6,16 & $-2,11$ & 1,42 \\
EMP/ETAB90 & 7,45 & 23,78 & 23,54 & 34,79 & 17,44 & 12,94 & 5,31 & 8,56 \\
EMP/ETAB99 & 6,85 & 17,87 & 17,81 & 25,38 & 18,44 & 11,76 & 5,04 & 8,04 \\
\hline
\end{tabular}

* Toutes les densités sont mesurées en $\mathrm{km}^{2}$.

Ces différents résultats mettent en évidence le caractère multipolarisé de la COMADI. Ils sont conformes dans les grandes lignes aux caractéristiques traditionnelles des centralités intraurbaines au sein des espaces urbains européens : au centre, densité et concentration de l'emploi vont de paire, tandis qu'en périphérie, ces deux termes ne sont plus synonymes. Néanmoins, quelques éléments suggèrent la possibilité d'une recomposition des centralités urbaines : la décroissance de l'emploi dans le centre historique et l'installation en périphérie d'établissements de tailles plus petites que par le passé. L'analyse des logiques de localisation des différentes activités économiques va nous permettre d'identifier plus précisément les mutations de la configuration urbaine au cours de la période.

\footnotetext{
${ }^{5} \mathrm{Si}$ on comptabilise, en revanche, la totalité de l'emploi : salarié, non salarié, public et privé, la COMADI gagne globalement des emplois sur cette période.
} 


\section{LA COMADI : UNE CONFIGURATION URBAINE EN MUTATION}

Trois phases de mutation des configurations urbaines sont généralement identifiées : celle déjà ancienne de l'étalement urbain liée à la suburbanisation de la population, celle de la création, à partir des années 1960, de pôles industriels et commerciaux périphériques et caractérisant la suburbanisation des emplois industriels et commerciaux et enfin, à partir des années 1980, celle de la formation de centres périphériques multifonctionnels liés à une phase de suburbanisation plus générale des emplois tertiaires (HARston et MUller, 1992 ; STANBACK, 1991). Plus que les précédents, ce mouvement de suburbanisation des emplois interroge sur les relations entre le centre historique et les centres périphériques car il conduit à la formation de pôles d'emplois périphériques attractifs pour les activités jusqu'alors considérées comme centrales telles que les activités de services supérieurs. Trois scénarios sont possibles : celui du déclin du CBD, celui au contraire d'une nouvelle forme de domination du centre par la spécialisation renforcée dans les fonctions stratégiques et internationales ou celui, enfin, d'une complémentarité entre pôle central et pôle périphérique. Plusieurs études comparatives montrent qu'aucun scénario ne domine actuellement (GASCHET, 2002 ; Shearmur et Coffey, 2002 ; Bingham et Kimble, 1995 ; Stanback, 1991). Dans ces conditions, il s'agit surtout de repérer l'histoire qui caractérise le mieux l'espace urbain étudié en analysant l'évolution de la composition économique des centres et l'importance du phénomène de suburbanisation des emplois de services supérieurs.

Différents éléments, caractérisant l'économie de l'agglomération dijonnaise, rendent potentiellement possible l'émergence de ces pôles périphériques multifonctionnels majeurs. Ainsi, aux cours des années 1980 et 1990, plusieurs parcs d'activités économiques, essentiellement tertiaires, ont été soit créés, soit étendus sur le territoire ${ }^{6}$. L'emploi tertiaire représente en 1999, 79,6\% de l'emploi total de la COMADI et 90\% de l'emploi tertiaire de l'aire urbaine dijonnaise ${ }^{7}$, confirmant ainsi le caractère fortement tertiaire et attractif de l'agglomération. Les services supérieurs représentent quant à eux $18 \%$ des emplois tertiaires de la COMADI. Par ailleurs, la diminution de l'emploi dans le pôle central et l'augmentation de l'emploi dans le pôle périphérique Nord au cours des années 1990, suggèrent un phénomène quantitatif de suburbanisation de l'emploi. L'étude de la composition sectorielle des pôles d'emplois et l'analyse de la structure de leur emploi tertiaire permettent alors d'apprécier de manière qualitative ce phénomène. Après avoir présenté les décompositions sectorielles utilisées (2.1.), l'étude de la composition économique des pôles d'emplois et de son évolution au cours des années 1990 (2.2.) va alors nous permettre de préciser quelles sont les principales mutations de la configuration urbaine de la COMADI (2.3.).

\subsection{Les décompositions sectorielles}

Disposant de données d'emplois répertoriées selon les nomenclatures NAP600 pour l'année 1990 et NAF700 pour l'année 1999, nous avons pu construire différents niveaux de désagrégation.

Une première décomposition en 7 secteurs d'activités ${ }^{8}$ nous permet de distinguer l'industrie (IND), la construction (CONST) et 5 secteurs d'activités tertiaires : le commerce de gros (COMGR), les infrastructures de transport et de communication (TRANS), les services aux particuliers (SPART), les activités financières, immobilières et d'assurance (FIA) et les

\footnotetext{
${ }^{6}$ Pour une surface totale d'environ 724 hectares (calcul au $1^{\text {er }}$ janvier 2001 d'après les documents fournis par l'Agence Intercommunale d'Urbanisme de l'Agglomération Dijonnaise).

${ }^{7}$ Chiffres calculés sur les données d'emploi au lieu de travail (RGP 1999, INSEE).

${ }^{8}$ Les emplois dans le secteur agricole et dans les administrations publiques n'ont pas été retenus compte tenu de l'incomplétude de la base de données SIRENE dans ces secteurs.
} 
services aux entreprises (SENT). Nous pouvons ainsi apprécier plus finement les logiques de localisation touchant par exemple les activités de bureaux (FIA, SENT), les activités nécessitant la proximité des consommateurs (SPART), des autres firmes (SENT) ou encore des moyens de transport et de communication (TRANS) de celles des activités à forte emprise foncière (IND ou CONST ou COMGR).

Une seconde décomposition nous permet par ailleurs de distinguer, d'une part, les activités tertiaires supérieures des autres activités tertiaires et de désagréger, d'autre part, le secteur des services supérieurs en différentes catégories. Plus précisément, nous considérons comme activités tertiaires supérieures les activités financières, immobilières et d'assurance et les services supérieurs aux entreprises. Même si l'ensemble des emplois du secteur FIA ne peuvent être considéré comme des emplois de services supérieurs, les décompositions d'activités fournies dans les nomenclatures disponibles ne permettent pas de les discerner des emplois FIA de services standard. Par ailleurs, dans ce secteur, nous trouvons à la fois des activités qui s'adressent aux particuliers et aux entreprises. En revanche, il est possible de distinguer dans le secteur SENT, les activités dites de service supérieur aux entreprises des activités de service aux entreprises standard. Ces dernières sont donc mises à part. Finalement et dans la mesure où le nombre d'emplois dans la COMADI était représentatif, la décomposition adoptée au sein des secteurs FIA et SENT est identique à celle retenue dans d'autres études (BoiteuX-Orain et Guillain, 2003 ; Alvergne et Shearmur, 1999). Au total, nous travaillons sur 11 types de services supérieurs : banques, assurances, auxiliaires de banques et assurances, immobilier, services techniques, R\&D, activités juridiques et comptables, publicité, architecture, ingénierie, conseil en gestion et administration. Cette décomposition doit nous aider à cerner d'éventuelles spécificités dans les logiques de localisation des activités tertiaires.

Enfin, nous avons également décomposé le secteur industriel afin d'examiner si certaines activités tertiaires tendent à se localiser près de certaines activités industrielles. La décomposition des activités industrielles que nous retenons est celle de la nomenclature NES36 de l'INSEE. Nous isolons alors 16 sous-secteurs industriels qui sont reconstruits, pour les données SIRENE de 1990 et 1999, à partir des nomenclatures d'activités NAP600 et NAF700. Les secteurs industriels concernés sont les suivants : industries agroalimentaires, 4 secteurs de fabrication de biens de consommation $(B C 1$ : habillement et cuir, $B C 2$ : édition, imprimerie et reproduction, $B C 3$ : pharmacie, parfumerie et entretien, $B C 4$ : équipement du foyer), industrie automobile, 3 secteurs de fabrication de biens d'équipement (BE1: construction navale, aéronautique et ferroviaire, BE2 : industries des équipements mécaniques, $B E 3$ : industrie des équipements électriques et électroniques), 6 secteurs de biens intermédiaires (BI1 : industries des produits minéraux, $B I 2$ : industrie textile, BI3 : industries du bois et du papier, BI4 : chimie, caoutchouc, plastiques, BI5 : métallurgie et transformation des métaux, BI6 : industrie des composants électriques et électroniques) et énergie.

La composition des secteurs tertiaires et industriels selon la nomenclature NAF700 est précisée en Annexe 3.

\subsection{La composition économique des pôles d'emplois dijonnais}

A l'aide de ces différentes données, nous avons pu analyser les combinaisons sectorielles des 3 principaux pôles d'emplois dijonnais et repérer leurs spécialisations en calculant des quotients de localisation. Les résultats sont présentés successivement pour le pôle central (cf. Tableau $\mathrm{n}^{\circ} 4$ ), le pôle périphérique Nord (cf. Tableau $\mathrm{n}^{\circ} 5$ ) et le pôle périphérique Sud (cf. Tableau $\mathrm{n}^{\circ} 6$ ). Dans chaque cas, nos commentaires concerneront déjà les grands secteurs de l'économie, puis les activités tertiaires et les activités de services supérieurs et enfin les activités industrielles. 
Le CBD est un pôle tertiaire par excellence : $88 \%$ des emplois en 1990, contre plus de $93 \%$ en 1999, relèvent globalement de ce secteur. $98 \%$ de cet emploi tertiaire appartient aux quatre secteurs des services aux particuliers, des services aux entreprises, des activités de transports et de communication et enfin du secteur Finance-Immobilier-Assurance.

Les grands secteurs tertiaires les plus représentés sont les services aux particuliers (plus du tiers des emplois du CBD) et les activités de transports et de communication (26\% en 1990 et près de $40 \%$ en 1999). Dans ce dernier cas, le CBD, notamment par la présence de la Gare et de la Poste centrale (Zone Grangier), concentre près de 50\% de l'emploi de la COMADI dans ce secteur. En 1990, les secteurs FIA et des services aux entreprises, au sein desquels on trouve les activités du tertiaire supérieur, représentent respectivement $12 \%$ et $13 \%$ de l'emploi du CBD, contre $11 \%$ et moins de $6 \%$ en 1999 . Néanmoins, en concentrant près de $50 \%$ de l'emploi de la COMADI dans le secteur FIA, le CBD est fortement spécialisé dans ce secteur tertiaire avec des quotients de localisation supérieurs à 2 pour les deux années. Pour les services aux entreprises, le CBD regroupe près de $29 \%$ des emplois de la COMADI en 1990 et seulement $10 \%$ en 1999. La spécialisation du pôle central dans le secteur des services aux entreprises est faible en 1990 (quotient de localisation=1,17) et n'existe plus en 1999 (quotient de localisation $=0,482$ ). Ces évolutions masquent des tendances différentes suivant le type de services considéré : service standard ou service supérieur.

Tableau $n^{\circ} 4$ : La structure sectorielle du CBD dijonnais

\begin{tabular}{|c|c|c|c|c|c|c|}
\hline & \multicolumn{3}{|c|}{1990} & \multicolumn{3}{|c|}{1999} \\
\hline & $\%$ CBD & QL & \% COMADI & $\%$ CBD & QL & $\%$ COMADI \\
\hline Construction & 1,2 & 0,114 & 2,8 & 0,9 & 0,092 & 2 \\
\hline \multirow{2}{*}{$\begin{array}{l}\text { Industrie } \\
\quad \text { Activités } \\
\text { Industrielles } \\
(Q L>1,4)\end{array}$} & 9 & 0,358 & 8,8 & 3,6 & 0,179 & 3,8 \\
\hline & Automobile & & & & & \\
\hline Tertiaire & 88 & 1,4 & 34,4 & 93,4 & 1,37 & 29,3 \\
\hline Transp\&Comm & 26,5 & 1,733 & 42,6 & 39,4 & 2,216 & 47,5 \\
\hline Serv Entrep. & 12,9 & 1,174 & 28,8 & 5,8 & 0,482 & 10,3 \\
\hline Serv. Part & 34 & 1,488 & 36,6 & 35,9 & 1,36 & 29,1 \\
\hline Com. Gros & 2,5 & 0,312 & 7,7 & 1,5 & 0,222 & 4,7 \\
\hline$F I A$ & 12,2 & 2,063 & 50,7 & 10,8 & 2,07 & 44,3 \\
\hline \multirow{2}{*}{$\begin{array}{l}\text { Services standard } \\
\text { Tertiaire supérieur }\end{array}$} & 7,8 & 1,249 & 30,7 & 1,9 & 0,4 & 8,6 \\
\hline & 17,3 & 1,625 & 39,9 & 14,6 & 1,186 & 25,4 \\
\hline $\begin{array}{l}\text { Services } \\
\text { Supérieurs } \\
(O L>1,4)\end{array}$ & \multicolumn{3}{|c|}{$\begin{array}{l}\text { Banques, Assurances, Auxil Bq et Ass } \\
\text { Immobilier, Act Jur et Comptable }\end{array}$} & \multicolumn{2}{|c|}{$\begin{array}{l}\text { Banques, Auxil Bq et Ass } \\
\text { Immobilier }\end{array}$} & \\
\hline
\end{tabular}

Ainsi, alors que le CBD concentrait près de $31 \%$ des activités de services standard aux entreprises en 1990, il n'en concentre plus que 8,6\% en 1999 et perd en conséquence sa spécialisation dans ces activités aux cours des années 1990. Si on s'intéresse maintenant aux seules activités de tertiaire supérieur, on remarque que le CBD concentre une part nettement moins importante des emplois de la COMADI dans ce secteur en $1999(25,4 \%)$ qu'en 1990 (40\%). La spécialisation du CBD dans les services supérieurs est néanmoins conservée sur la période, mais elle s'affaiblit : le quotient de localisation est de 1,625 en 1990 et de 1,186 en 1999. Par activités, les spécialisations les plus fortes concernent sur les deux années les activités de banques, d'auxiliaires de banques et d'assurances et l'immobilier. En revanche, les spécialisations dans le secteur de l'assurance et des services juridiques et comptables en 1990 disparaissent en 1999. La faiblesse de la représentation du secteur industriel dans le CBD ne permet pas de repérer des spécialisations particulières par sous secteurs, sauf le cas particulier, en 1990, du secteur Automobile, qui s'explique par l'existence dans le quartier Arsenal d'une 
usine de fabrication de pièces automobiles, qui quitta ensuite ce quartier central. Dans ces conditions, nous ne pouvons induire de liens particuliers entre les localisations tertiaires et industrielles au centre de l'agglomération dijonnaise.

\section{Analyse des pôles périphériques}

Le pôle périphérique Nord reste spécialisé d'étude dans les secteurs de la construction et de l'industrie, même si on observe un affaiblissement de cette spécialisation sur la période. En effet, ces deux secteurs représentent respectivement $16 \%$ et $40,6 \%$ de l'emploi du pôle en 1990 contre près de $15 \%$ et seulement $24,8 \%$ en 1999 . Environ $26 \%$ de l'emploi industriel de la COMADI est concentré dans ce pôle aussi bien en 1990 qu'en 1999, tandis que l'emploi de l'agglomération dans le secteur de la construction voit sa concentration augmenter de 1990 à 1999 , passant de $24,7 \%$ à $32,5 \%$. Ce pôle périphérique n'est spécialisé ni dans le tertiaire, ni dans le tertiaire supérieur bien que l'on observe une augmentation sensible des quotients de localisation aux cours des années 90 .

Tableau $n^{\circ} 5$ : La structure sectorielle du Pôle périphérique Nord

\begin{tabular}{|c|c|c|c|c|c|c|}
\hline & \multicolumn{3}{|c|}{1990} & \multicolumn{3}{|c|}{1999} \\
\hline & $\%$ PNord & $\mathbf{Q L}$ & $\%$ COMADI & \% PNord & $\mathbf{Q L}$ & $\%$ COMADI \\
\hline Construction & 16,1 & 1,526 & 24,6 & 14,7 & 1,551 & 32,5 \\
\hline Industrie & 40,6 & 1,623 & 26,2 & 24,8 & 1,236 & 25,9 \\
\hline $\begin{array}{l}\text { Activités } \\
\text { Industrielles } \\
(Q L>1,4)\end{array}$ & \multicolumn{3}{|c|}{$\begin{array}{l}\text { IAA, Pharmacie, parfumerie et entretien } \\
\text { Const. navale, aéronautique et ferroviaire, } \\
\text { Ind. des équip. mécaniques, } \\
\text { Industries des produits minéraux } \\
\text { Ind. des comp. électriques et électroniques }\end{array}$} & \multicolumn{3}{|c|}{$\begin{array}{l}\text { Pharmacie, parfumerie et entretien } \\
\text { Ind. des équip. mécaniques, } \\
\text { Industries des produits minéraux } \\
\text { Ind. des comp. électriques et électroniques }\end{array}$} \\
\hline Tertiaire & 43,1 & 0,685 & 11,1 & 59,9 & 0,879 & 18,4 \\
\hline Transp\&Comm & 5,4 & 0,353 & 5,7 & 8 & 0,449 & 9,4 \\
\hline Serv. Entrep. & 6,9 & 0,627 & 10,1 & 10,7 & 0,894 & 18,7 \\
\hline Serv. Part & 14,3 & 0,628 & 10,1 & 26,6 & 1,005 & 21,1 \\
\hline Com. Gros & 15,1 & 1,92 & 31 & 12,3 & 1,825 & 38,3 \\
\hline$F I A$ & 1,3 & 0,225 & 3,6 & 2,3 & 0,445 & 9,3 \\
\hline Services standard & 3,1 & 0,505 & 8,1 & 2,8 & 0,567 & 11,9 \\
\hline Tertiaire supérieur & 5,1 & 0,475 & 7,7 & 10,3 & 0,835 & 17,5 \\
\hline $\begin{array}{l}\text { Services } \\
\text { Supérieurs } \\
(Q L>1,4)\end{array}$ & \multicolumn{3}{|c|}{$\begin{array}{l}\text { Services Techniques } \\
\text { Conseil }\end{array}$} & \multicolumn{3}{|c|}{$\begin{array}{l}\text { Services Techniques } \\
\text { Conseil, Ingénierie }\end{array}$} \\
\hline
\end{tabular}

Si on analyse la décomposition des activités tertiaires, on remarque que le pôle périphérique Nord est fortement spécialisé dans le commerce de gros en 1990 et en 1999 et que la part des activités de services aux particuliers représente 26,5\% de son emploi en 1999 contre seulement $14,3 \%$ en $1990.21 \%$ des activités de services aux particuliers de la COMADI se situent dans ce pôle périphérique, chiffre qui se rapproche de celui observé pour le CBD (29\%). Pour les services aux entreprises, alors que le CBD concentre seulement $10 \%$ des emplois de la COMADI dans ce secteur en 1999, le pôle Nord en concentre près de $19 \%$, ce qui constitue une augmentation notable par rapport à 1990 (10\%). Cette tendance est opposée à celle observée pour le CBD (29\% en 1990). Environ $12 \%$ des services standard aux entreprises de la COMADI se localisent dans le Pôle périphérique Nord en 1999, mais cela représente une faible part de la structure d'emplois du pôle $(2,8 \%)$. Pour les services supérieurs, les plus fortes spécialisations concernent, sur les deux années, les services techniques et les activités de conseil, et pour 1999, les activités d'ingénierie. Ces spécialisations sont à rapprocher des activités industrielles en général. Plus précisément, le pôle périphérique Nord est fortement spécialisé, sur la période d'étude, dans les activités de 
production de biens de consommation (pharmacie, parfumerie et entretien), de biens d'équipement mécanique et de biens intermédiaires (produits minéraux et composants électriques et électroniques).

Le pôle périphérique Sud présente des caractéristiques générales similaires à celles observées pour le pôle périphérique Nord. Il est spécialisé dans le secteur de la construction et dans l'industrie et ce n'est pas un pôle tertiaire. Cependant, si on analyse plus finement les activités tertiaires on remarque que ce pôle est, comme son homologue, spécialisé dans les activités de commerce de gros, mais qu'il est également spécialisé dans les activités de transport et de communication. La représentation des services aux particuliers est stable sur la période et assez modeste : 13\% des emplois de la COMADI. Concernant les activités de services aux entreprises, on observe une très forte augmentation de la représentation de ce secteur entre 1990 et 1999 : de $2,8 \%$ à $11 \%$, ce qui représentait 5,2\% des emplois de la COMADI en 1990 contre $18 \%$ en 1999 . Cette dernière part est similaire à celle du pôle périphérique Nord et est supérieure à celle du CBD. Cette augmentation se traduit naturellement par une augmentation de la représentation des activités de services supérieurs dans la périphérie sud de l'agglomération dijonnaise. Alors qu'aucune spécialisation dans de telles activités n'était observée en 1990, le pôle périphérique Sud devient en 1999 spécialisé très fortement dans les activités de R\&D (quotient de localisation de 4,3) et plus modestement dans les activités de conseil (quotient de localisation de 1,4). Enfin, si on s'intéresse aux activités industrielles, on note, de très fortes spécialisations dans de nombreuses industries avec un resserrement, en 1999, autour des industries de fabrication de biens d'équipement et de biens intermédiaires.

Tableau $n^{\circ} 6$ : La structure sectorielle du Pôle périphérique Sud

\begin{tabular}{|c|c|c|c|c|c|c|}
\hline & \multicolumn{3}{|c|}{1990} & \multicolumn{3}{|c|}{1999} \\
\hline & $\%$ PSud & QL & $\%$ COMADI & $\%$ PSud & QL & $\%$ COMADI \\
\hline Construction & 11,3 & 1,068 & 22 & 12,2 & 1,288 & 25 \\
\hline Industrie & 36 & 1,439 & 29,6 & 29,9 & 1,489 & 28,9 \\
\hline $\begin{array}{l}\text { Activités } \\
\text { Industrielles } \\
(Q L>1,4)\end{array}$ & $\begin{array}{l}\text { Pharmacie, } p \\
\text { Equipement d } \\
\text { Const. navale } \\
\text { Ind. des équip } \\
\text { Ind. des équip } \\
\text { Industries du } \\
\text { Chimie, caout } \\
\text { Métallurgie e }\end{array}$ & $\begin{array}{l}\text { fumerie et e } \\
\text { foyer } \\
\text { aéronautiqu } \\
\text { mécaniques } \\
\text { électriques } \\
\text { is et du pap } \\
\text { houc, plastic } \\
\text { ransformati }\end{array}$ & $\begin{array}{l}\text { ntretien } \\
\text { et ferroviaire } \\
\text { t électroniques } \\
\text { ier } \\
\text { ues } \\
\text { on des métaux }\end{array}$ & $\begin{array}{l}\text { Automobile } \\
\text { Const. navale } \\
\text { Ind. des équip } \\
\text { Industrie text } \\
\text { Industries du } \\
\text { Chimie, caout } \\
\text { Ind. des comp }\end{array}$ & $\begin{array}{l}\text { aéronautiq } \\
\text { mécaniques } \\
\text { ois et du pa } \\
\text { houc, plasti } \\
\text { électriques }\end{array}$ & $\begin{array}{l}\text { e et ferroviaire } \\
\text { ier } \\
\text { ues } \\
\text { télectroniques }\end{array}$ \\
\hline Tertiaire & 52 & 0,827 & 17 & 57 & 0,836 & 16,2 \\
\hline Transp\&Comm & 24,1 & 1,578 & 32,5 & 18 & 1,015 & 19,7 \\
\hline Serv Entrep. & 2,8 & 0,253 & 5,2 & 11,1 & 0,925 & 17,9 \\
\hline Serv. Part & 14,4 & 0,629 & 12,9 & 18 & 0,678 & 13,1 \\
\hline Com. Gros & 10,4 & 1,31 & 27 & 9,7 & 1,442 & 27,9 \\
\hline$F I A$ & 0,4 & 0,071 & 1,5 & 0,2 & 0,032 & 0,6 \\
\hline Services standard & 1,3 & 0,215 & 4,4 & 4,1 & 0,84 & 16,3 \\
\hline $\begin{array}{l}\text { Tertiaire supérieur } \\
\quad \text { Services } \\
\text { Supérieurs } \\
(Q L>1,4)\end{array}$ & 1,9 & 0,174 & 3,6 & $\begin{array}{l}\text { R\&D } \\
\text { Conseil }\end{array}$ & 0,581 & 11,3 \\
\hline
\end{tabular}

\subsection{Une configuration urbaine issue de logiques traditionnelles et contemporaines}

Ces différents résultats montrent que la configuration spatiale de l'emploi au sein de la COMADI est à la fois caractérisée par des logiques de localisation traditionnelles et 
contemporaines selon les types d'activités économiques. Ces évolutions peuvent être sectorielles, si elles associent différents types d'activités, et géographiques, lorsqu'elles se manifestent par une suburbanisation de l'emploi.

Les logiques traditionnelles sont celles fondées par exemple sur la proximité géographique entre la population et les activités de services aux particuliers, entre les activités FIA et la population ou les autres activités économiques. Le pôle d'emploi central, dont on a mis en évidence le caractère mixte, satisfait alors plus facilement cette fonction. De même, les établissements de petite taille, comme ceux des secteurs FIA et les services aux entreprises et certains types de services aux particuliers, peuvent se localiser dans les quartiers centraux, alors que les activités plus consommatrices d'espace, comme l'industrie, la construction et le commerce de gros, se localiseront dans les Zones d'Activités Economiques des périphéries.

Ces logiques traditionnelles sont observées au sein de la COMADI, mais elles se modifient au cours de la période empruntant certains traits caractéristiques des logiques contemporaines de localisation des emplois tertiaires observés sur d'autres espaces urbains. En effet, nous pouvons observer quatre évolutions importantes (cf. Tableau $\mathrm{n}^{\circ} 7$ ).

Tableau $n^{\circ} 7$ : La suburbanisation des emplois dans la COMADI

\begin{tabular}{|c|c|c|c|c|c|c|c|}
\hline Pôles & CBD & Pnord & Psud & Périph $^{(a)}$ & Pôles ${ }^{(b)}$ & hors Pôles & COMADI \\
\hline$\triangle \mathrm{EMP}$ & $\begin{array}{r}-3113 \\
(-17,04)\end{array}$ & $\begin{array}{r}2824 \\
(23,52)\end{array}$ & $\begin{array}{r}-1590 \\
(-10,39)\end{array}$ & $\begin{array}{r}1234 \\
(4,52)\end{array}$ & $\begin{array}{l}-1669 \\
(-3,49)\end{array}$ & $\begin{array}{l}-1894 \\
(-7,16)\end{array}$ & $\begin{array}{l}-3563 \\
(-4,79)\end{array}$ \\
\hline$\triangle \mathrm{CONST}$ & $\begin{array}{r}-87 \\
(-39,55)\end{array}$ & $\begin{array}{r}247 \\
(12,74)\end{array}$ & $\begin{array}{r}-51 \\
(-2,95)\end{array}$ & $\begin{array}{r}196 \\
(5,34)\end{array}$ & $\begin{array}{r}113 \\
(2,67)\end{array}$ & $\begin{array}{r}-1255 \\
(-34,46)\end{array}$ & $\begin{array}{r}-1142 \\
(-14,52)\end{array}$ \\
\hline$\Delta \mathrm{IND}$ & $\begin{array}{r}-1094 \\
(-66,75\end{array}$ & $\begin{array}{r}-1204 \\
(-24,68)\end{array}$ & $\begin{array}{r}-1420 \\
(-25,76)\end{array}$ & $\begin{array}{r}-2624 \\
(-25,25)\end{array}$ & $\begin{array}{r}-3789 \\
(-28,56)\end{array}$ & $\begin{array}{r}-633 \\
(-11,85)\end{array}$ & $\begin{array}{r}-4422 \\
(-23,76)\end{array}$ \\
\hline$\triangle$ TERT & $\begin{array}{l}-1935 \\
(-12,03\end{array}$ & $\begin{array}{r}3710 \\
(71,64)\end{array}$ & $\begin{array}{r}-149 \\
(-1,87)\end{array}$ & $\begin{array}{r}3561 \\
(27,10)\end{array}$ & $\begin{array}{r}1903 \\
(6,35)\end{array}$ & $\begin{array}{r}-440 \\
(-2,61)\end{array}$ & $\begin{array}{r}1463 \\
(3,13)\end{array}$ \\
\hline$\triangle T R A N S$ & $\begin{array}{r}1133 \\
(23,44)\end{array}$ & $\begin{array}{r}536 \\
(82,84)\end{array}$ & $\begin{array}{r}-1214 \\
(-32,93)\end{array}$ & $\begin{array}{r}-678 \\
(-15,64)\end{array}$ & $\begin{array}{r}736 \\
(7,95)\end{array}$ & $\begin{array}{r}487 \\
(23,26)\end{array}$ & $\begin{array}{r}1223 \\
(10,77)\end{array}$ \\
\hline$\triangle S E N T$ & $\begin{array}{r}-1479 \\
(-62,78)\end{array}$ & $\begin{array}{r}766 \\
(92,62)\end{array}$ & $\begin{array}{r}1097 \\
(257,51)\end{array}$ & $\begin{array}{r}1863 \\
(148,68)\end{array}$ & $\begin{array}{r}560 \\
(15,14)\end{array}$ & $\begin{array}{r}-227 \\
(-5,08)\end{array}$ & $\begin{array}{r}333 \\
(4,08)\end{array}$ \\
\hline$\triangle S P A R T$ & $\begin{array}{r}-768 \\
(-12,36)\end{array}$ & $\begin{array}{r}2217 \\
(128,67)\end{array}$ & $\begin{array}{r}257 \\
(11,68)\end{array}$ & $\begin{array}{r}2474 \\
(63,06)\end{array}$ & $\begin{array}{r}1575 \\
(14,96)\end{array}$ & $\begin{array}{r}140 \\
(2,17)\end{array}$ & $\begin{array}{r}1715 \\
(10,09)\end{array}$ \\
\hline$\triangle C O M G R$ & $\begin{array}{r}-224 \\
(-49,67)\end{array}$ & $\begin{array}{r}7 \\
(0,38)\end{array}$ & $\begin{array}{r}-248 \\
(-15,66)\end{array}$ & $\begin{array}{r}-241 \\
(-7,08)\end{array}$ & $\begin{array}{r}-515 \\
(-12,84)\end{array}$ & $\begin{array}{r}-579 \\
(-31,08)\end{array}$ & $\begin{array}{r}-1094 \\
(-18,62)\end{array}$ \\
\hline$\triangle F I A$ & $\begin{array}{r}-597 \\
(-26,75)\end{array}$ & $\begin{array}{r}184 \\
(115,00)\end{array}$ & $\begin{array}{r}-41 \\
(-64,06)\end{array}$ & $\begin{array}{r}143 \\
(63,84)\end{array}$ & $\begin{array}{r}-453 \\
(-18,44)\end{array}$ & $\begin{array}{r}-261 \\
(-13,41)\end{array}$ & $\begin{array}{r}-714 \\
(-16,22)\end{array}$ \\
\hline $\begin{array}{l}\Delta \text { Services } \\
\text { Standard }\end{array}$ & $\begin{array}{r}-1133 \\
(-79,23)\end{array}$ & $\begin{array}{r}32 \\
(8,42)\end{array}$ & $\begin{array}{r}359 \\
(174,27)\end{array}$ & $\begin{array}{r}391 \\
(66,72)\end{array}$ & $\begin{array}{r}-754 \\
(-35,96)\end{array}$ & $\begin{array}{r}-436 \\
(-17,01)\end{array}$ & $\begin{array}{r}-1190 \\
(-25,54)\end{array}$ \\
\hline $\begin{array}{l}\Delta \text { Services } \\
\text { Supérieurs }\end{array}$ & $\begin{array}{r}-943 \\
(-29,86) \\
\end{array}$ & $\begin{array}{r}918 \\
(151,23) \\
\end{array}$ & $\begin{array}{r}697 \\
(245,42) \\
\end{array}$ & $\begin{array}{r}1615 \\
(181,26) \\
\end{array}$ & $\begin{array}{r}861 \\
(21,21) \\
\end{array}$ & $\begin{array}{r}-52 \\
(-1,35) \\
\end{array}$ & $\begin{array}{r}809 \\
(10,23) \\
\end{array}$ \\
\hline
\end{tabular}

(a) Pôle Nord et Pôle Sud.

(b) CBD, Pôle Nord, Pôle Sud, Pôle Isolé Est et Pôle Isolé Nord.

Note : entre parenthèses figurent les variations en pourcentage.

La première touche les activités tertiaires en général, les activités de services supérieurs et les services standard aux entreprises en particulier qui se localisaient davantage au centre en 1990, et qui se localisent majoritairement dans les pôles périphériques en 1999. Ceux-ci concentrent dorénavant $32,6 \%$ des activités tertiaires, $28,2 \%$ des activités de services standard et $28,8 \%$ des services supérieurs de la COMADI contre respectivement $29,3 \%, 8,6 \%$ et $25,4 \%$ pour le CBD. Cette tendance est à rapprocher de l'évolution des emplois sur la COMADI 
entre 1990 et 1999 : alors que le nombre d'emplois dans les service supérieurs aux entreprises augmente, le CBD perd ces types d'emplois et même si le nombre d'emplois dans les activités FIA ou de services standard aux entreprises diminue, la périphérie gagne ces types d'emplois. L'évolution globale de la structure économique des emplois est alors fortement marquée géographiquement au sein de l'agglomération. La seconde évolution montre que les activités de services aux entreprises sont plus touchées par cette tendance car le secteur FIA reste une spécialisation forte du CBD. La troisième, liée aux précédentes, touche alors les logiques de localisation des établissements de petites tailles : on s'aperçoit par exemple, que le nombre moyen d'emplois par établissement dans le pôle périphérique Nord diminue entre 1990 et 1999, alors que ce pôle gagne globalement près de 3000 emplois. Ainsi, "préférence pour la centralité et contrainte foncière" n'entrent plus nécessairement de la même manière dans les arbitrages de localisation de ces établissements.

Enfin, la dernière évolution montre une différence de structure industrielle et tertiaire entre les deux pôles périphériques. Le pôle périphérique Nord semble aller vers une tertiarisation accrue de sa structure d'emplois car il présente des variations d'emplois positives dans chaque secteur tertiaire et sur la majorité des secteurs de services supérieurs. Cette évolution est à relier au démarrage et l'achèvement, aux cours des années 90 , d'une nouvelle zone d'activités économiques (la Toison d'Or-D62) au sein du pôle Nord. Le pôle périphérique Sud, qui perd globalement des emplois au cours de la période, gagne cependant des emplois dans les secteurs clés de l'économie contemporaine : les services supérieurs aux entreprises. Une construction multifonctionnelle est associée au pôle périphérique Nord tandis qu'une restructuration fonctionnelle liée à l'industrie est plus caractéristique du pôle périphérique Sud.

Comment peut-on alors décrire, au vu de ces différents constats, "l'histoire" de la recomposition urbaine de l'agglomération dijonnaise ? Si quantitativement le CBD perd des emplois tertiaires et des emplois de services supérieurs, il concentre toujours près du tiers des activités tertiaires de la COMADI et reste fortement spécialisé dans les activités stratégiques du secteur FIA. Si les pôles périphériques gagnent globalement des emplois (+1 234 emplois) et des emplois tertiaires (+3561) c'est surtout le fait du pôle périphérique Nord. En revanche, la suburbanisation des emplois de services aux entreprises semble davantage se faire au sein du pôle périphérique Sud où l'on observe une augmentation et une croissance plus fortes du nombre de ces emplois. Les activités de services supérieurs tendent également à se suburbaniser. Les gains ou les pertes d'emplois selon les secteurs d'activités, au sein des pôles périphériques, conduisent par ailleurs à une structure plus diversifiée de leur composition sectorielle. En conséquence, des mutations sectorielles se mettent en place au sein des pôles suite aux changements de logiques de localisation des activités économiques. Ces mutations modifient la nature des relations entre le centre et la périphérie de deux manières : une complémentarité fonctionnelle entre les différents pôles suivant les spécialisations de chacun d'entre eux et une relative substituabilité entre le CBD et le pôle périphérique Nord.

Cette image de la structure spatiale et économique de la COMADI reste cependant dépendante de la méthode d'identification des pôles que nous avons appliquée. En particulier, si on regarde les parts des emplois de la COMADI présents dans ces pôles en 1999 et selon les catégories d'activités (cf. Tableau $\mathrm{n}^{\circ} 8$ ), on s'aperçoit que nous répertorions $50 \%$ de l'emploi des services aux entreprises, $54 \%$ de l'emploi dans le secteur FIA et $56 \%$ de l'emploi des services supérieurs. 
Tableau $n^{\circ} 8$ : Répartition de l'emploi dans et en dehors des pôles

\begin{tabular}{|c|c|c|c|c|c|c|c|c|c|c|}
\hline Pôles & EMP & CONST & IND & TERT & TRANS & SENT & SPART & COMGR FIA & SorvSta & ervSup \\
\hline & 6 & & 1,28 & 64,03 & & 45,30 & 61,97 & $68,2955,79$ & 4. & 51,32 \\
\hline$\%$ COMADI99 & 65,30 & 64,51 & 66,79 & 66,03 & 79,47 & 50,12 & 64,71 & $73,1454,31$ & 38,7 & 56,43 \\
\hline
\end{tabular}

Ce constat peut signifier qu'il existe un biais dans notre analyse et que nous avons assimilé l'évolution de la configuration urbaine de la COMADI aux seules évolutions de l'emploi localisé dans les pôles. Dans cet esprit, nous n'avons capté que ce qui se passe dans les pôles d'emplois, négligeant une dimension possible des mutations spatiales : la formation de pôles d'emplois tertiaires ou la diffusion de ces emplois sur l'ensemble du territoire. C'est ce que nous allons maintenant étudier.

\section{POLARITÉS INTRA-URBAINES ET LOCALISATION DES SERVICES SUPÉRIEURS}

La tertiarisation de l'agglomération dijonnaise se manifeste de manière quantitative et qualitative. Quantitativement déjà, la part de l'emploi tertiaire dans l'emploi total de la COMADI passe de 62,94\% en 1990 à 67,75\% $1999^{9}$. Qualitativement ensuite car, dans plus de $75 \%$ des 114 zones étudiées, les activités tertiaires sont présentes à plus de $50 \%$. On remarque aussi que la répartition géographique de l'emploi tertiaire est plus ou moins diffuse ou concentrée selon les catégories car elle dépend de facteurs ou de contraintes de localisation propres à chaque catégorie. Ainsi, les services aux particuliers sont présents en majorité ${ }^{10}$ dans 55\% des 114 zones de la COMADI, ce qui est le reflet d'une recherche de proximité à la population. Les activités des secteurs du commerce de gros et des transports et communication sont nettement plus concentrées car elles sont présentes en majorité dans respectivement $22 \%$ et $18 \%$ des 114 zones : leurs logiques de localisation sont fortement liées à des sites particuliers comme les Zones d'Activités Economiques ou à la proximité des grandes infrastructures de transport. Les activités du secteur FIA sont également assez concentrées et figurent en majorité dans $26 \%$ des 114 zones de la COMADI et enfin les services aux entreprises sont présents en majorité dans $41 \%$ des cas.

Ces deux derniers secteurs qui alimentent les activités de services supérieurs ne sont recensés dans les pôles d'emplois de la COMADI qu'à une faible majorité et obéissent à des logiques de localisation en mutation. La manière dont ils occupent l'espace dijonnais et contribuent à l'évolution de la configuration urbaine au cours des années 1990 est approfondie dans cette section. Après avoir rappelé quelques principes généraux sur les modes de localisation des activités de services supérieurs (3.1.), nous analysons la configuration spatiale de ces emplois au sein de la COMADI (3.2.) et indiquons comment l'évolution de leurs logiques de localisation influence les mutations urbaines de l'agglomération (3.3.).

\subsection{Des logiques de localisation complexes}

Les choix de localisation des activités de services supérieurs résultent d'arbitrages souvent complexes, quelquefois encore mal cernés. Ces arbitrages concernent majoritairement les facteurs de localisation suivants : l'emprise foncière qui est souvent celle des activités de

\footnotetext{
${ }^{9}$ Rappelons qu'il s'agit ici uniquement de l'emploi mesuré par les effectifs salariés du secteur privé et pour les 5 grands secteurs sur lesquels nous travaillons : sont ainsi exclus les effectifs des services publics et les effectifs non salariés des activités de service.

${ }^{10}$ Plus précisément, les commentaires s'appuient ici sur le nombre de zones qui possèdent un quotient de localisation supérieur à 1 pour le secteur concerné : l'idée de "majorité" est donc relative à la part de ce secteur dans la structure globale de l'emploi de la COMADI.
} 
bureaux, la qualité des aménagements de ces bureaux notamment en matière de NTIC, la proximité des grandes infrastructures de transport pour l'efficacité des relations physiques avec l'extérieur (déplacements vers les clients, déplacement de la main d'œuvre...), le besoin de proximité géographique avec la clientèle, avec les fournisseurs et/ou avec les activités de même type et/ou avec les activités différentes afin de bénéficier d'externalités informationnelles, la préférence pour la centralité qui traduit en fait la recherche de sites prestigieux et valorisants tels que les centres-villes, sièges des activités de décisions et de pouvoirs économiques et politiques (COFFEY et SHEARMUR, 2002 ; COFFEY, DrOLET, POLÈSE, 1996 ; LONGCORE et REES, 1996). Face à cette complexité, les modèles de la microéconomie urbaine restent encore assez limités (BOITEUX-ORAIN et HURIOT, 2002) et ont du mal à rendre compte de la diversité des logiques de localisation observées suivant les types de services supérieur concernés. A partir d'études empiriques sur l'Ile-de-France (BOITEUX-ORAIN et GUILlAIN, 2003 ; ALVERGNE et SHEARMUR, 1999) ou sur l'agglomération lyonnaise (AGUILÉRA, 2002), quatre schémas de localisation des activités de services supérieurs peuvent être dégagés : le maintien d'une localisation centrale, le choix d'une localisation périphérique dans des pôles existants, le choix d'une localisation périphérique dans des nouveaux pôles et enfin, le choix d'une localisation périphérique diffuse calquée sur une logique résidentielle. Ces différents schémas, suivant le type et le nombre des activités concernées, produisent et font évoluer les configurations urbaines et il est donc important de pouvoir les identifier. Par exemple, si les activités de services supérieurs considérées comme des activités centrales, ont tendance à se localiser de plus en plus massivement en périphérie, alors le CBD perd de son influence économique ou se spécialise dans certaines seulement de ces activités. Si le phénomène de suburbanisation des activités tertiaires supérieures suit une logique résidentielle diffuse, alors aucun pôle périphérique n'émerge. Si les activités de services supérieurs renforcent et diversifient les pôles périphériques existants, alors on peut observer la formation de Edges Cities.

C'est en gardant à l'esprit ces différents éléments que nous allons décrire les schémas d'organisation des emplois de services supérieurs dans la COMADI.

\subsection{La configuration spatiale des emplois de services supérieurs dans la COMADI}

Pour identifier les tendances de localisation des emplois de services supérieurs, nous avons choisi d'analyser l'ensemble des zones qui concentre, en $1999,75 \%$ de l'emploi de la COMADI dans les activités FIA et les services aux entreprises ${ }^{11}$. Ce critère nous conduit à retenir 27 zones qui possèdent, dans ces secteurs, au moins 160 emplois (cf. Tableau ${ }^{\circ} 9$ ). Dans cette sélection, toutes les zones sauf 3 (Daix, Neuilly-lès-Dijon et la Zone d'Activités Economiques de Talant) soit appartiennent aux pôles d'emplois de la COMADI (14 zones) soit leur sont directement contiguës (cf. Carte $\mathrm{n}^{\circ} 3$ ). Plus précisément, $95 \%$ du tertiaire supérieur des pôles d'emplois est présent dans cette sélection si bien que nous retrouverons les éléments soulignés dans la section précédente. Cependant, nous pouvons, grâce à cette sélection, analyser les logiques de localisation de 30\% d'emplois de services supérieurs supplémentaires. Nous remarquons alors que $18 \%$ des services supérieurs de la COMADI se localisent directement à côté des pôles d'emplois.

\footnotetext{
${ }^{11}$ L'analyse de la localisation des différentes activités est faite suivant la décomposition fournie en annexe 3. Nous pouvons ainsi analyser, non seulement, les services supérieurs FIA et les services supérieurs aux entreprises, mais également les services standard aux entreprises.
} 


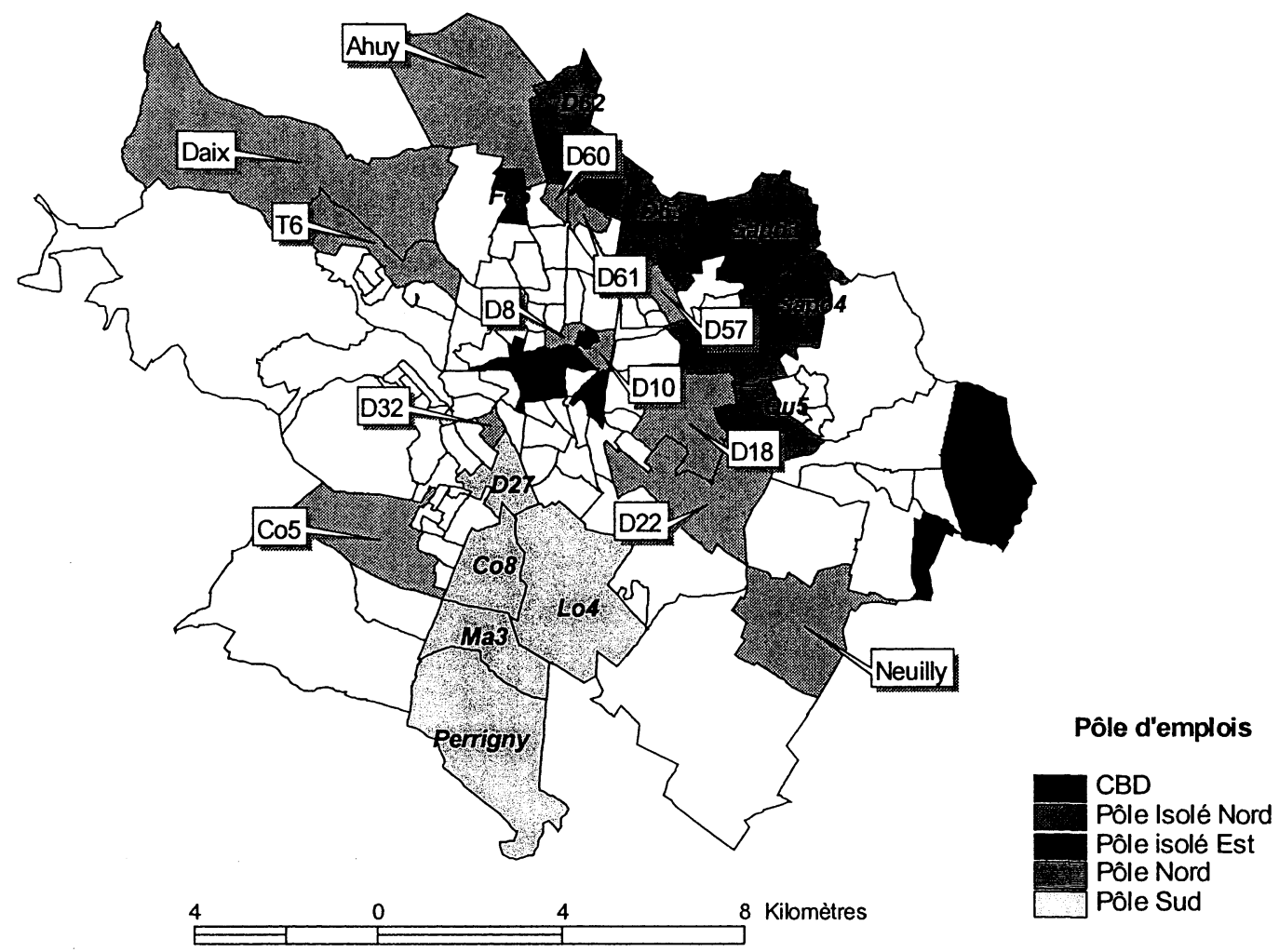

On remarque également que $5 \%$ de l'emploi tertiaire supérieur présent dans cette sélection occupe des zones particulières comme les communes résidentielles de Daix ou de Neuilly-lèsDijon, ou la ZAE de Talant donnant ainsi une impression de diffusion des activités de services supérieurs au sein de la COMADI sur toute la partie Nord-Est de la couronne périphérique.

Trois traits importants ressortent alors de ces premiers éléments (cf. Tableau n ${ }^{\circ} 9$ ). En premier lieu, les logiques de localisation des services supérieurs dans la COMADI sont fortement liées à la présence ou à la proximité des pôles d'emplois. En deuxième lieu, près de deux tiers des services supérieurs privilégient dans cette sélection une occupation périphérique plutôt que centrale.

Enfin, en troisième lieu, une logique résidentielle de localisation des services apparaît dans les communes périphériques résidentielles : qu'elle concerne plutôt les services supérieurs (commune de Daix) ou les services standard aux entreprises (commune de Neuilly-lès-Dijon) voire les deux types de services (commune d'Ahuy).

On remarque par ailleurs que les zones qui possèdent des ratio "emploi supérieur / emploi tertiaire supérieur" les plus importants (supérieurs à 60\%), se situent en périphérie Nord et Est, et sont, à l'exception de la zone d'activités Sud-Est de Saint-Apollinaire, des zones contiguës ou extensives des pôles existants. Enfin, on peut noter que la croissance de l'emploi sur la période est très inégale selon les zones. Si on excepte la zone Concorde (D60) qui n'existait pas en 1990, on peut constater que dans 14 zones, l'emploi régresse contre 12 dans lequel il s'accroît. Concernant l'emploi des services supérieurs, il augmente globalement de près de $10 \%$ entre 1990 et 1999 pour la COMADI, alors qu'il croît de près de $32 \%$ pour les 27 zones. On remarque cependant des évolutions contrastées selon la localisation de ces zones : 
Tableau $n^{\circ} 9$ : Caractéristiques des zones de services supérieurs

\begin{tabular}{|c|c|c|c|c|c|c|c|c|c|c|}
\hline Zone & Type & $\begin{array}{c}\text { Emploi } \\
1999\end{array}$ & $\begin{array}{c}\text { Tertiaire } \\
1999\end{array}$ & $\begin{array}{l}\text { Serv. } \\
\text { Sup. } \\
1999 \\
\end{array}$ & $\begin{array}{c}\text { Serv. } \\
\text { Stand. } \\
1999 \\
\end{array}$ & $\begin{array}{c}\text { Ratio } \\
\text { Sup/Tert }\end{array}$ & $\begin{array}{c}\Delta \text { Empl. } \\
(\%)\end{array}$ & $\begin{array}{c}\Delta \text { Tertiaire } \\
(\%)\end{array}$ & $\begin{array}{c}\Delta \text { Serv. } \\
\text { Sup. } \\
(\%) \\
\end{array}$ & $\begin{array}{c}\Delta \text { Serv. } \\
\text { Stand. } \\
(\%)\end{array}$ \\
\hline \multicolumn{11}{|c|}{$C B D$} \\
\hline D01 & Pôle & 1501 & 1444 & 276 & 44 & 19,11 & $-28,32$ & $-25,49$ & $-14,29$ & $-74,57$ \\
\hline D02 & Pôle & 1048 & 946 & 251 & 2 & 26,53 & $-23,34$ & $-23,28$ & $-57,67$ & $-60,00$ \\
\hline D04 & Pôle & 4080 & 3906 & 937 & 43 & 23,99 & $-5,12$ & $-4,36$ & $-21,66$ & $-83,20$ \\
\hline D06 & Pôle & 1200 & 1130 & 379 & 75 & 33,54 & $-44,52$ & $-43,67$ & $-1,81$ & $-90,85$ \\
\hline D08 & Contiguë & 747 & 664 & 339 & 38 & 51,05 & $-35,66$ & $-32,79$ & $-29,67$ & $-79,68$ \\
\hline D09 & Pôle & 1164 & 1070 & 162 & 35 & 15,14 & 48,66 & 52,64 & $-27,03$ & 52,17 \\
\hline D10 & Contiguë & 584 & 445 & 105 & 55 & 23,60 & $-45,88$ & -52 & $-63,03$ & $-82,76$ \\
\hline \multicolumn{11}{|c|}{ Périphérie Nord } \\
\hline Daix & Extension & 793 & 376 & 335 & 0 & 89,10 & 104,38 & 1346,15 & 16650 & -100 \\
\hline Ta6 & Extension & 633 & 433 & 128 & 95 & 29,56 & $-10,85$ & $-4,2$ & $-11,11$ & 39,71 \\
\hline Fo5 & Pôle & 1088 & 462 & 192 & 26 & 41,56 & 15,5 & $-2,33$ & 1820 & $-13,33$ \\
\hline Ahuy & Contiguë & 518 & 384 & 68 & 142 & 17,71 & 36,68 & 41,18 & 1033,33 & $-32,70$ \\
\hline D60 & Contiguë & 635 & 563 & 389 & 87 & 69,09 & & & & \\
\hline D61 & Contiguë & 463 & 436 & 372 & 50 & 85,32 & 72,76 & 62,69 & 45,88 & 284,62 \\
\hline D62 & Pôle & 2670 & 2551 & 461 & 160 & 18,07 & 284,73 & 626,78 & 184,57 & \\
\hline D63 & Pôle & 5558 & 2478 & 199 & 112 & 8,03 & 3,75 & 17,16 & 298,00 & $-52,74$ \\
\hline \multicolumn{11}{|c|}{ Périphérie Est } \\
\hline D57 & Contiguë & 431 & 342 & 2 & 226 & 0,58 & $-51,84$ & $-42,9$ & $-98,15$ & $-34,87$ \\
\hline Sapo3 & Pôle & 1406 & 800 & 156 & 37 & 19,50 & $-12,13$ & 9,89 & $-11,86$ & 94,74 \\
\hline Sapo4 & Pôle & 1184 & 277 & 263 & 6 & 94,95 & $-7,79$ & 54,75 & 1653,33 & 50,00 \\
\hline D18 & Contiguë & 817 & 337 & 175 & 39 & 51,93 & $-5,55$ & 10,86 & 3,55 & 77,27 \\
\hline $\mathrm{D} 22$ & Contiguë & 492 & 326 & 7 & 178 & 2,15 & $-14,43$ & 25,38 & $-58,82$ & 474,19 \\
\hline Qu5 & Pôle & 4014 & 2783 & 446 & 97 & 16,03 & 30,62 & 54,1 & 119,70 & $-19,17$ \\
\hline Neuilly & Extension & 370 & 247 & 2 & 168 & 0,81 & $-15,14$ & 43,6 & 100 & 112,66 \\
\hline \multicolumn{11}{|c|}{ Périphérie Sud } \\
\hline D32 & Contiguë & 725 & 664 & 27 & 264 & 4,07 & 3,57 & 5,73 & 50 & $-19,02$ \\
\hline Co8 & Pôle & 4776 & 3278 & 676 & 295 & 20,62 & $-8,38$ & 2,28 & 755,70 & 180,95 \\
\hline Co5 & Contiguë & 352 & 312 & 58 & 114 & 18,59 & 238,46 & 428,81 & 114,81 & \\
\hline Ma3 & Pôle & 1505 & 1070 & 60 & 193 & 5,61 & 41,85 & 57,12 & 1400 & 2657,14 \\
\hline Lo4 & Pôle & 5259 & 2085 & 207 & 36 & 9,93 & 0,46 & 70,9 & 60,47 & 350 \\
\hline \multicolumn{2}{|c|}{ Total 27 Zones } & 44013 & 29809 & 6672 & 2617 & 22,38 & $-3,01$ & 16,1 & 31,83 & $-23,30$ \\
\hline \multicolumn{2}{|c|}{ COMADI } & 70770 & 48249 & 8718 & 3470 & 18,07 & $-4,79$ & 3,13 & 10,23 & $-25,54$ \\
\hline
\end{tabular}

l'emploi des services supérieurs diminue pour toutes les zones centrales, tandis qu'il augmente dans la majorité des zones périphériques et souvent de manière spectaculaire (Daix, Fo5, D61, Ahuy, Sapo4 ou Ma3 ou, par exemple) du fait de la quasi absence de ce type d'emplois en 1990 ; les zones nouvellement identifiées ou celles appartenant aux pôles existants étant indifféremment concernées. Ces évolutions sont aussi à mettre en parallèle avec celles des services standard aux entreprises : globalement, ce type de services est en chute d'environ $25 \%$ dans la COMADI et dans les zones sélectionnées. Souvent, les zones périphériques gagnent des emplois de services supérieurs mais perdent des emplois de services standard. On trouve néanmoins quelques zones qui accroissent de manière significative leur emploi dans 
les deux types de services (D61, D62 ou Co8, par exemple). On assiste finalement à des mutations de logiques de localisation des activités de services, en général, et des activités de services supérieurs, en particulier, au sein de la COMADI.

\subsection{Des logiques de localisation des activités de services supérieurs en mutation}

$\mathrm{Si}$ on regarde plus précisément les caractéristiques sectorielles de ces zones, plusieurs informations complémentaires apparaissent qui permettent de distinguer les comportements de localisation selon le type d'activités (cf. Tableau $\left.{ }^{\circ} 10\right)$.

Nous constatons de manière nette la spécialisation des zones centrales dans les activités de services supérieurs liées au secteur FIA et dans les activités juridiques et comptables. Ce résultat confirme, pour ces activités dédiées aussi bien aux ménages qu'aux entreprises, l'attractivité du pôle d'emplois central que nous avions déjà constatée. Il renforce aussi cette image du fait de l'addition de deux zones contiguës (D8 et D10) proches de la nouvelle cité judiciaire.

Les logiques de localisation dans les zones périphériques apparaissent plus hétéroclites, comme cela se constate dans d'autres études empiriques menées sur l'Ile-de-France (BOITEUXORAIN et GUILlaIN, 2003 ; AlVERGNE et SHEARMUR, 1999) ou sur l'agglomération lyonnaise (AGUILÉRA, 2002), par exemple. Ainsi ces logiques peuvent s'expliquer par des choix résidentiels de localisation propres aux dirigeants qui implantent leurs sociétés sur leur lieu de résidence, ce qui entraîne par la même une diffusion des activités de services supérieurs dans les zones résidentielles non centrales : Ahuy, Daix, Neuilly-lès-Dijon, les quartiers dijonnais de l'Université (D18) ou Concorde (D60) ou encore du Vieux Bourg (Co5) à Chenôve. Dans les autres cas, les implantations se font dans les Zones d'Activités Economiques, la recherche d'un environnement économique lisible étant alors privilégiée : l'image attractive des pôles d'emplois périphériques constatée précédemment se renforce alors par l'addition de zones fortement spécialisées dans des services supérieurs diversifiés (Banques, Assurance, Ingénierie, Services Techniques, Publicité) dans le pôle périphérique Nord ou dans des services supérieurs orientés vers les entreprises (Services Techniques, Ingénierie, R\&D) dans le pôle périphérique Sud.

Si on rapproche ces résultats de l'évolution de l'emploi dans les pôles, on peut mettre en avant deux tendances. Etant donnée la décroissance de l'emploi dans le CBD, on peut supposer que sa spécialisation se fait par fuite des autres emplois de services supérieurs et que cette tendance touche également les zones contiguës au CBD. Par opposition, la croissance de l'emploi de services supérieurs dans les pôles périphériques semble avoir deux effets très différents. Dans la périphérie Nord-Est de la COMADI, on assiste à la formation d'un pôle multifonctionnel grâce notamment aux effets des récentes politiques d'aménagement de zones tertiaires d'excellence, aux cours des années 1990, dans les quartiers Concorde (D60) et Clos de Pouilly (D61). Dans la périphérie Sud, au contraire, se renforce l'image d'un pôle composé de zones d'activités plus anciennes et plutôt dédiées aux activités industrielles.

Toutes proportions gardées, on retrouve ainsi au sein de la COMADI, les mêmes évolutions dans les logiques de localisation des services supérieurs que celles observées dans des agglomérations nettement plus grandes. Ces évolutions sont suffisamment fortes pour modifier la configuration urbaine dijonnaise, celle-ci passant d'une structure multipolarisée dominée par le CBD à une structure toujours multipolarisée mais où le pôle central se spécialise tandis que la périphérie se diversifie. 
Tableau n`10: Spécialisation des zones d'emplois de services supérieurs

\begin{tabular}{|c|c|c|c|}
\hline Nom & Type & Spécialisation 1999* & Spécialisation 1990* \\
\hline \multicolumn{4}{|l|}{ Centre } \\
\hline D01 & Existant & Banq, Aux & Assu, Banq \\
\hline D02 & Existant & Archi, Jur\&Comp, Immob, Aux & Assu, Jur\&Comp, Conseils \\
\hline D04 & Existant & Immo, Banq & Banq, Immo, \\
\hline D06 & Existant & Immo, Aux, Banq & Aux, Autres \\
\hline D08 & Contiguë & Jur\&Comp, Banq, Archi & Aux, Banq \\
\hline D09 & Existant & Jur\&Comp, Archi & Archi, Jur\&Comp, Aux, Assu \\
\hline D10 & Contiguë & Jur\&Comp, Ingé, Aux & Assu, Autres \\
\hline \multicolumn{4}{|c|}{ Périphérique Nord } \\
\hline Daix & Extension & Pub & Ingé \\
\hline Ta6 & Extension & Archi, Jur\&Comp, Autres & Archi, Jur\&Comp \\
\hline Fo5 & Existant & ST & Pub, Autres \\
\hline Ahuy & Contiguë & Archi, Autres, Pub & Autres \\
\hline D60 & Contiguë & Assur & \\
\hline D61 & Contiguë & Banq & Banq, R\&D \\
\hline D62 & Existant & Ingé, ST, Jur\&Comp & ST, Ingé, Jur\&Comp \\
\hline D63 & Existant & Ingé, ST & Autres \\
\hline \multicolumn{4}{|c|}{ Périphérique Est } \\
\hline D57 & Contiguë & Autres & ST, Autres, Ingé \\
\hline Sapo3 & Existant & Pub, Conseil, Ingé, ST & Conseils, ST \\
\hline Sapo4 & Existant & Conseils & ST \\
\hline D18 & Contiguë & R\&D, ST, Ingé, Conseils & R\&D, ST, Ingé, Conseils \\
\hline D22 & Contiguë & Autres & Archi, Aux, Autres \\
\hline Qu5 & Existant & Banq, Ingé & Conseils, Ingé, Banq \\
\hline Neuilly & Extension & Autres & Autres \\
\hline \multicolumn{4}{|c|}{ Périphérique Sud } \\
\hline D32 & Contiguë & Autres & Autres \\
\hline $\operatorname{Co5}$ & Contiguë & Ingé, Autres & Ingé, Jur\&Comp \\
\hline $\operatorname{Co} 8$ & Existant & $\mathrm{R} \& \mathrm{D}$ & R\&D, Pub, Conseils, Archi, Autres \\
\hline $\mathrm{Ma3}$ & Existant & Autres, ST & Pub, Immo, Autres \\
\hline Lo4 & Existant & ST, Ingé & ST, Ingé \\
\hline
\end{tabular}

Note : les abréviations suivantes sont retenues: Archi (architecture), Autres (autres services aux entreprises), Aux (auxiliaires de banques et assurances), Banq (banques), Conseils (conseils en gestion et administration), Immo (immobilier), Ingé (ingénierie), Jur\&Comp (services juridiques et comptables), Pub (publicité), R\&D (recherche et développement), ST (services techniques).

* seulement pour les activités présentant un quotient de localisation supérieur à 1,4 et classées par ordre décroissant.

\section{CONCLUSION}

On peut considérer aujourd'hui comme des faits bien établis, les mutations des configurations urbaines dans les grandes aires métropolitaines et le rôle important joué par les services supérieurs dans ce processus. Notre étude de l'agglomération dijonnaise montre que ces faits peuvent également caractériser une unité urbaine, certes de taille moyenne à l'échelle française, mais de petite taille à l'échelle occidentale. La COMADI s'engage ainsi aux cours des années 1990 dans un processus de spécialisation de son centre-ville dans les activités de services supérieurs du secteur Finance-Immobilier-Assurance, spécialisation qui s'opère essentiellement par une perte de spécialisation dans les autres catégories de services. Au 
contraire, se forme dans la partie Nord-Est de l'agglomération dijonnaise, un pôle d'emplois multi-fonctionnels, attractif, où se concentrent des activités industrielles, des activités tertiaires et des activités de services supérieurs.

Cependant, au delà de ces constats globaux d'évolution, il semble aujourd'hui plus difficile d'établir si un modèle unique de restructuration des pôles centraux et des pôles périphériques s'applique et ceci indépendamment de la taille des unités urbaines. Ce que l'on observe, c'est au contraire, une diversité des caractéristiques sectorielles des pôles périphériques. Là encore, l'agglomération dijonnaise n'échappe pas à cette observation. Bien que l'on n'ait identifié que deux pôles d'emplois périphériques, ils possèdent des structures différentes : au pôle multifonctionnel du Nord s'oppose, au Sud, un pôle d'emplois de taille similaire plutôt industriel et attirant des activités de services supérieurs liées au secteur industriel.

Ces résultats conduisent à envisager deux types de politiques urbaines d'aménagement : la création de parcs tertiaires d'excellence à proximité des anciennes zones d'activités économiques pour accompagner les phénomènes de suburbanisation des activités de services supérieurs, d'une part, et l'aménagement de cités des affaires dans le centre-ville pour en préserver l'attractivité et le symbolisme, d'autre part.

\section{BIBLIOGRAPHIE}

AgulLÉRA A., 2002, "Services aux entreprises, centralité et multipolarisation. Le cas de Lyon ", Revue d'Economie Régionale et Urbaine, n³, pp. 397-422.

Alvergne C., SHEarmur R., 1999, « Nouvelles centralités, nouvelles périphéries en Ile-deFrance : les changements de logiques de localisation des services supérieurs "), in GollaIN V., SAllez A. (eds.), Emploi et territoires en Ile-de-France : Prospectives, Paris, Editions de l'Aube.

BAumont C., Bourdon F., 2002, «Centres secondaires et recomposition économique des espaces urbains. Le cas de la Communauté de l'Agglomération Dijonnaise $(1990 ; 1999)$ », Document de travail, LATEC, Université de Bourgogne, n²002-04.

Baumont C., Le Gallo J., 2000, «Les nouvelles centralités urbaines », in BAUMONT C., COMBES P.-P., DERYCKE P.-H., JAYET H. (eds.), Economie géographique: les théories à l'épreuve des faits, Paris, Economica (Bibliothèque de Science Régionale), pp. 211-239.

Baumont C., ERTUR C., Le Gallo J., 2003, Spatial Analysis of Employment and Population Density: The Case of the Agglomeration of Dijon, 1999, Geographical Analysis, à paraître.

BINGHAM R.D., KIMBLE D., 1995, "The Industrial Composition of Edge Cities and Downtowns: The New Urban Reality », Economic Development Quarterly, Vol.9, n³ (August 1995), pp.259-272.

BoiteuX-OraIN C., GuIllaIN R., 2003, «Changes in the Intra-Metropolitan Location of Producer Services in Ile-De-France (1978-1997): Do Information Technologie Promote a more Dispersed Spatial Pattern ", Document de travail, LATEC, Université de Bourgogne, $\mathrm{n}^{\circ} 2003-06$.

BoIteUX-OraIN C., HuRIOT J.-M., 2002, « Modéliser la suburbanisation. Succès et limites de la microéconomie urbaine », Revue d'Economie Régionale et Urbaine, $\mathrm{n}^{\circ} 1, \mathrm{pp}$. 73-104.

Coffey W.J., PolÈSE M., Drolet R., 1996, « Examining the Thesis of Central Business District Decline : Evidence from the Montreal Metropolitan Area ", Environment and Planning $A$, vol. 28, pp. 1795-1814. 
COFFEY W.J., Shearmur R., 2002, «Agglomeration and dispersion service employment in the Montreal metropolitan region, 1981-1996», Urban Studies, 39, 3, pp. 359-378.

ForSTALl R.L. and GREENE R.P., 1997, « Defining job concentrations: the Los Angeles case », Urban Geography, 18, pp. 705-739.

FuJita M., OGawa H., 1982, "Multiple Equilibria and Structural Transition of Nonmonocentric Urban Configurations ", Regional Science and Urban Economics, vol. 12, pp. 161-196.

Garreau J., 1991, Edge City: Life on the New Frontier, New York: Anchor Books, Doubledey.

GASCHET F., 2000, «La structure d'un espace urbain polycentrique : les pôles d'activités de la métropole bordelaise », in DERYCKE P.-H. (ed), "Structure des villes, entreprises et marchés urbains », L'Harmattan, Paris, pp. 95-126.

GASCHET F., 2002, "The New Intra-Urban Dynamics: Suburbanisation and Functional Specialisation in French Cities », Papers in Regional Science, vol. 81, n 1, pp. 63-81.

GiUliano G., Small K.A., 1991, "Subcenters in the Los Angeles Region », Regional Science and Urban Economics, vol. 21, pp. 163-182.

Harston T.A., MUller P.O., 1992, «The Suburban Downtown and Urban Economic Development ", in MiLls E.S., MCDonald, J.F. (Eds), Sources of Metropolitan Growth, New Brunswick, NJ: Center for Urban Policy Research, pp. 147-158.

INSEE, 2000, "IRIS-2000 : un nouveau découpage pour mieux lire la ville », Chiffres pour l'Alsace, $\mathrm{n}^{\circ} 43, \mathrm{p} .5$.

LE JEANNIC T., 1998, «L'élaboration du zonage en aires urbaines (ZAU) » in les Découpages du territoire (dixièmes entretiens Jacques Cartiers), Insee Méthodes, n076-77-78, pp. 71-90.

LONGCORE, T.R. and REES, P.W., 1996, " Information technology and downtown restructuring: The case of New York City's financial district ", Urban Geography, Vol. 17, pp. 354-372.

MCDONALD J.F., 1987, "The Identification of Urban Subcenters ", Journal of Urban Economics, 21, pp. 242-258.

SHEARMUR R., COFFEY W.J., 2002, «A Tale of Four Cities: Intrametropolitan Employment Distribution in Toronto, Montreal, Vancouver and Ottawo-Hull, 1981-1996 ", Environment and Planning A, Vol. 34, pp. 575-598.

STANBACK T.M., 1991, The New Suburbanisation, Boulder: Westview Press. 


\section{ANNEXE $n^{\circ} 1:$ Le zonage IRIS $2000^{\circledR}$}

L'appellation IRIS signifie Ilots Regroupés pour l'Information Statistique.

L'IRIS-2000 $^{\circledR}$ est un découpage infra-communal disponible pour toutes les communes urbaines d'au moins 10000 habitants et la plupart des communes de 5000 à 10000 habitants (16 000 IRIS-2000 ${ }^{\circledR}$ en France, dont 15400 en métropole). C'est un " petit quartier ", qui se définit comme un ensemble d'îlots (pâtés de maisons) contigus. Les IRIS-2000 ${ }^{\circledR}$ se déclinent en trois types de zones (INSEE, 2000) :

- IRIS d'habitat : IRIS-2000 ${ }^{\circledR}$ dont la population se situe entre 1800 et 5000 habitants. Ils sont homogènes quant au type d'habitat,

- IRIS d'activité : IRIS-2000 ${ }^{\circledR}$ qui regroupent plus de 1000 salariés et comptent deux fois plus d'emploi salarié que de population résidente,

- IRIS divers : IRIS-2000 ${ }^{\circledR}$ de superficie importante, et à un usage particulier (bois, parcs, zones portuaires...).

Dans les bases de données couvrant plusieurs communes, les données IRIS correspondront alors soit à l'IRIS-2000 ${ }^{\circledR}$ pour les communes découpées, soit à la commune pour les petites communes non découpées.

ANNEXE $n^{\circ} 3$ : La décomposition sectorielle des services et de l'industrie

\begin{tabular}{|c|c|}
\hline \multicolumn{2}{|c|}{ Services supérieurs } \\
\hline Nom & NAF700 \\
\hline Banques & $\begin{array}{l}651 \mathrm{~A}, 651 \mathrm{C} \text { à } 651 \mathrm{~F}, \\
652 \mathrm{~A}, 652 \mathrm{C}, 652 \mathrm{E}, 652 \mathrm{~F}\end{array}$ \\
\hline Assurances & $\begin{array}{l}660 \mathrm{~A}, \quad 660 \mathrm{C}, \quad 660 \mathrm{E} \\
660 \mathrm{G}\end{array}$ \\
\hline $\begin{array}{l}\text { Auxiliaires de banques } \\
\text { et assurances }\end{array}$ & $671 \mathrm{~A}, 671 \mathrm{C}, 671 \mathrm{E}, 672 \mathrm{Z}$ \\
\hline Immobilier & $\begin{array}{l}701 \mathrm{~A} \text { à } 701 \mathrm{D}, 701 \mathrm{~F}, \\
702 \mathrm{~A} \text { a } 702 \mathrm{C}, 703 \mathrm{~A}, \\
703 \mathrm{C} \text { à } 703 \mathrm{E}\end{array}$ \\
\hline Services techniques & $\begin{array}{l}721 \mathrm{Z}, 722 \mathrm{Z}, 723 \mathrm{Z}, 724 \mathrm{Z}, \\
725 \mathrm{Z}, 741 \mathrm{E}\end{array}$ \\
\hline R\&D & $731 Z, 732 Z$ \\
\hline $\begin{array}{l}\text { Activités juridiques } \\
\text { et techniques }\end{array}$ & $741 \mathrm{~A}, 741 \mathrm{C}$ \\
\hline Publicité & $744 \mathrm{~A}, 744 \mathrm{~B}$ \\
\hline Architecture & $742 A, 742 B$ \\
\hline Ingénierie & $742 \mathrm{C}$ \\
\hline $\begin{array}{l}\text { Conseils en gestion } \\
\text { et administration }\end{array}$ & $741 \mathrm{G}, 741 \mathrm{~J}$ \\
\hline \multicolumn{2}{|c|}{ Autres services aux entreprises } \\
\hline & $\begin{array}{l}\text { 745A, 745B, 711Z, 712A, } \\
\text { 712C, 712E, 713A, 713C, } \\
\text { 713E, 713G, 714A, 714B, } \\
\text { 725Z, 726Z, 743A, 743B, } \\
\text { 746Z, 747Z, 748A, 748B, } \\
\text { 748D, 748F, 748G, 748J, } \\
748 \mathrm{~K}\end{array}$ \\
\hline
\end{tabular}

\begin{tabular}{|c|c|}
\hline \multicolumn{2}{|l|}{ Industrie } \\
\hline Nom & NAF60 ou NAF700 \\
\hline IAA & $15 ; 16$ \\
\hline $\begin{array}{l}\text { Industries liées à } \\
\text { l'habillement }\end{array}$ & $17,18,19$ \\
\hline $\begin{array}{l}\text { Industries liées au } \\
\text { bois }\end{array}$ & 20,21 \\
\hline $\begin{array}{l}\text { Industries liées à } \\
\text { l'édition }\end{array}$ & 22 \\
\hline $\begin{array}{l}\text { Industries liées aux } \\
\text { matières premières }\end{array}$ & $24,25,26$ \\
\hline $\begin{array}{l}\text { Industries liées à la } \\
\text { métallurgie }\end{array}$ & 27,28 \\
\hline $\begin{array}{l}\text { Fabrication } \\
\text { d'équipements et de } \\
\text { matériels }\end{array}$ & $29,30,31$ \\
\hline $\begin{array}{l}\text { Fabrication } \\
\text { d'appareils de } \\
\text { communication et } \\
\text { de précision }\end{array}$ & 32,33 \\
\hline $\begin{array}{ll}\text { Fabrication } & \text { de } \\
\text { matériel } & \text { de } \\
\text { transport } & \end{array}$ & 34,35 \\
\hline Ameublement & 361 \\
\hline Industries diverses & $\begin{array}{l}10,11,12,13,14 \\
\text { Autres que } 361 \\
37,40,41\end{array}$ \\
\hline
\end{tabular}




\begin{tabular}{|c|c|}
\hline NOM de II ZONG URBAINE & Code \\
\hline AHUY & Ahuy \\
\hline PISCINE - VALENDONS & Col \\
\hline PETIGNYS - CHAUFFERIE & $\mathrm{Co} 2$ \\
\hline CHAPITRE - BIBLIOTHEQUE & $\mathrm{Co} 3$ \\
\hline SAINT EXUPERY & $\mathrm{Co} 4$ \\
\hline VIEUX BOURG - GRANDS CRUS & $\mathrm{Co5}$ \\
\hline ATELIERS SNCF & $\mathrm{Co6}$ \\
\hline MAIRIE - STADE & $\mathrm{Co} 7$ \\
\hline ZONE INDUSTRIELLE & $\mathrm{Co} 8$ \\
\hline STRD & $\mathrm{Co} 9$ \\
\hline BREUIL - FLEURS & Ch1 \\
\hline CORCELLES - BAS DE CHANOT & $\mathrm{Ch} 2$ \\
\hline CENTRE VILLE - CLOS ST SAUVEUR & $\mathrm{Ch} 3$ \\
\hline CHATEAU - VISITATION & $\mathrm{Ch} 4$ \\
\hline ZONE ECONOMIQUE & $\mathrm{Ch} 5$ \\
\hline DAIX & Daix \\
\hline MONGE & D1 \\
\hline CORDELIERS & D2 \\
\hline SAINT MICHEL & D3 \\
\hline GRANGIER & D4 \\
\hline J J ROUSSEAU & D5 \\
\hline DARCY & D6 \\
\hline LES ROSES & D7 \\
\hline REPUBLIQUE & D8 \\
\hline CLEMENCEAU & D9 \\
\hline DAVOUT & D10 \\
\hline PETIT CITEAUX & D11 \\
\hline SAINT PIERRE & $\mathrm{D} 12$ \\
\hline DOCTEUR LAVALLE & D13 \\
\hline VOLTAIRE & D14 \\
\hline LYAUTEY & D15 \\
\hline PARC DES SPORTS & D16 \\
\hline CHAMPMAILLOT & D17 \\
\hline UNIVERSITES & D18 \\
\hline LENTILLERES & D19 \\
\hline PETITES ROCHES & D20 \\
\hline MANSART & D21 \\
\hline ABATTOIRS & D22 \\
\hline CASTEL & D23 \\
\hline STEARINERIE & D24 \\
\hline CARROUSEL & D25 \\
\hline GREUZE & D26 \\
\hline ARSENAL & D27 \\
\hline BEL AIR & D28 \\
\hline LARREY & D29 \\
\hline BOURROCHES OUEST & D30 \\
\hline BOURROCHES EST & D31 \\
\hline TROIS FORGERONS & D32 \\
\hline LES VALENDONS & D33 \\
\hline LA MONTAGNE & D34 \\
\hline TIRE PESSEAU & D35 \\
\hline LE LAC & D36 \\
\hline EDOUARD BELIN & D37 \\
\hline CHAMP PERDRIX & D38 \\
\hline CHARTREUSE & D39 \\
\hline ARQUEBUSE & D40 \\
\hline TANNERIES & D41 \\
\hline PROVIDENCE & D42 \\
\hline CARRIERES BACQUIN & D43 \\
\hline FRANCOIS POMPON & D44 \\
\hline LES HAUTS DE MONTCHAPET & D45 \\
\hline EUGENE SPULLER & D46 \\
\hline LA CHARMETTE & D47 \\
\hline
\end{tabular}

\begin{tabular}{|c|c|}
\hline NOM de la 20 NE URBAINE & Code \\
\hline FAUCONNET & $\mathrm{D} 48$ \\
\hline JOUVENCE OUEST & D49 \\
\hline JOUVENCE EST & D50 \\
\hline BALZAC & D51 \\
\hline STALINGRAD & D52 \\
\hline CASERNES & D53 \\
\hline SACRE COEUR & D54 \\
\hline YORK & D55 \\
\hline LOCHERES & D56 \\
\hline GRESILLES CENTRE & D57 \\
\hline CASTELNAU & D58 \\
\hline CHARLES DE GAULLE & D59 \\
\hline CONCORDE & D60 \\
\hline CLOS DE POUILLY & D61 \\
\hline LA TOISON D OR & D62 \\
\hline Z I NORD EST & D63 \\
\hline LA GARE & D64 \\
\hline LE BOCAGE & D65 \\
\hline COMBE A LA SERPENT LE LAC & D66 \\
\hline VIEUX VILLAGE & Fol \\
\hline SAVERNEY & Fo2 \\
\hline SAINT MARTIN & Fo3 \\
\hline MAJNONI & Fo4 \\
\hline ACTIVITES ECONOMIQUES & Fo5 \\
\hline BIEF DU MOULIN & Lo1 \\
\hline BOURG-VALENTIN-PREVOTS & Lo2 \\
\hline PARC POUSSOTS-POMMERETS & Lo3 \\
\hline ZONE INDUSTRIELLE-COLOMBIERE & Lo4 \\
\hline LE BOURG & Mal \\
\hline CHAMPAGNE HAUTE & $\mathrm{Ma} 2$ \\
\hline ZAC & $\mathrm{Ma} 3$ \\
\hline NEUILLY-LES-DIJON & Neuilly \\
\hline OUGES & Ouges \\
\hline PERRIGNY-LES-DIJON & Perry \\
\hline PLOMBIERES-LES-DIJON & Plomb \\
\hline ATRIAS-VIEUX VILLAGE-GRAND CHAIGNET & Qu1 \\
\hline LA FONTAINE AUX JARDINS & Qu3 \\
\hline LES HUCHES & Qu3 \\
\hline PLACE CENTRALE-QUETIGNEROTS-PRE BOURGEOT & Qu4 \\
\hline ZA EUROPE-CAP VERT-GRAND MARCHE & Qu5 \\
\hline NORD VILLAGE & Sapo1 \\
\hline SUD VILLAGE & Sapo2 \\
\hline NORD EST & Sapo3 \\
\hline SUD EST & Sapo4 \\
\hline SENNECEY-LES-DIJON & Sennec \\
\hline QUARTIER 01 & Tal \\
\hline QUARTIER 02 & $\mathrm{Ta} 2$ \\
\hline QUARTIER 03 & $\mathrm{Ta} 3$ \\
\hline QUARTIER 04 & $\mathrm{Ta} 4$ \\
\hline QUARTIER 05 & $\mathrm{Ta} 5$ \\
\hline QUARTIER 06 & $\mathrm{Ta} 6$ \\
\hline & \\
\hline & \\
\hline & \\
\hline & \\
\hline & \\
\hline & \\
\hline & \\
\hline & \\
\hline & \\
\hline & \\
\hline & \\
\hline
\end{tabular}

